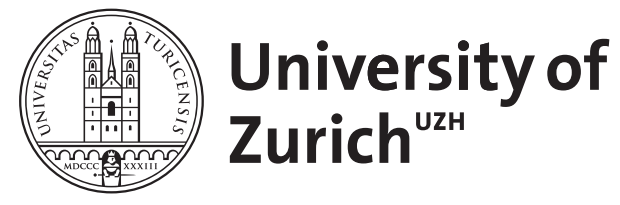

Zurich Open Repository and Archive

University of Zurich

University Library

Strickhofstrasse 39

CH-8057 Zurich

www.zora.uzh.ch

Year: 1995

Le double décomposé. Rencontres des vivants et des morts à la fin du Moyen Age

Kiening, Christian

Posted at the Zurich Open Repository and Archive, University of Zurich

ZORA URL: https://doi.org/10.5167/uzh-113630

Journal Article

Published Version

Originally published at:

Kiening, Christian (1995). Le double décomposé. Rencontres des vivants et des morts à la fin du Moyen Age. Annales. Histoire, Sciences sociales, 50(5):1157-1190. 


\section{LE DOUBLE DÉCOMPOSÉ Rencontres des vivants et des morts à la fin du Moyen Age}

Christian KIENING

\section{La multiplication des cadavres}

En 1538, les frères Gaspar et Melchior Trechsel publièrent à Lyon Les simulachres \& historiees faces de la mort, avtant elegamment pourtraictes, que artificiellement imaginées : un nouveau type de danse macabre, rapidement fameux grâce aux gravures sur bois dessinées plus d'une décennie avant par Hans Holbein le Jeune et gravées par Hans Lützelburger ${ }^{1}$. L'édition, qui donnait de fortes impulsions à la figuration et la méditation de la mort des siècles suivants ${ }^{2}$, est précédée par une épître dédicatoire, due à l'écrivain lyonnais Jean de Vauzelles («dun vray Zele»; 1495-avant 1559) qui s'occupa beaucoup d'œuvres charitables'. Il s'y adresse à l'abbesse du couvent Saint-Pierre-les-Nonnains de Lyon, Jeanne de Thouzelles («Touszele »), et définit la fonction des images de la mort en tant que «le vray, \&

* Je tiens à remercier vivement Alain Boureau de m'avoir donné l'occasion de présenter l'essentiel de cet article dans le cadre de son séminaire de l'EHESS, Paris.

1. Fac-similés: The Dance of Death by Hans Holbein the Younger. A Complete Facsimile of the Original 1538 Edition of «Les simulachres \& historiees faces de la mort». With a New Introduction by W. L. Gundersheimer, New York, 1971; La Danse Macabre de Hans Holbein 1538. Introduction de P. AHNNE, Paris, 1970 (sans les «Figures de la mort, moralement descriptes \& peintes... »; $\left.\mathrm{Hi}^{\mathrm{r}} \mathrm{ss}\right)$.

2. Répertoire bibliographique des éditions jusqu'au 19e siècle : H. F. MAsSManN, Literatur der Totentänze [d'abord une collection des articles dans Serapeum, 1840-1850], Hildesheim, 1961, pp. 5-59; G. FrAnz, Huberinus - Rhegius - Holbein. Bibliographische und druckgeschichtliche Untersuchung der verbreitetsten Trost- und Erbauungsschriften des 16. Jahrhunderts, Nieuwkoop, 1973 , pp. 38-45, 130-144. Sur les images, toujours le plus exhaustif, A. GoetTe, Holbeins Totentanz und seine Vorbilder, Strasbourg, 1897, pp. 192-276; en outre St. KOZÁKY, Der Totentanz von heute, Budapest, 1941, pp. 100-112.

3. L. DE VAuzelles, « Notice sur Jean de Vauzelles, prieur de Montrottier, littérateur et poète lyonnais du $16^{\mathrm{e}}$ siècle ", Revue du Lyonnais, $3^{\mathrm{e}}$ série, 13, 1872, pp. 52-73; voir aussi les articles de R. BARroux, Dictionnaire des lettres françaises. Le $16^{\mathrm{e}}$ siècle, G. Grente éd. et al., Paris, 1951, p. 685 ; A. CiorAnEsCO, Bibliographie de la littérature française $d u 16^{e}$ siècle, Paris, 1959 , p. 680 ss. 


\section{IMAGES DE LA MORT, DESTRUCTION DES IMAGES AU MOYEN AGE}

propre miroer auquel on doibt corriger les defformitez de peché » (Aiiv ${ }^{4}$. Il reprend le motif du miroir, répandu dans le contexte du memento mori et contemptus mundi ${ }^{5}$, mais, en renvoyant aux peintures et monuments (" spirituelz miroers ») « mis a lentree des Eglises, \& Cymitieres [...] pour veoir si entre ces ossemens des mortz pourroit trouuer aulcune difference des riches, $\&$ des pouures » (ibid.), Vauzelles lui donne un aspect concrètement visuel. Un peu plus loin il pose explicitement la question de la représentation figurée de la mort :

Quelle figure de Mort peult estre par viuant representee ? Ou, comment en peuuent deuiser ceulx, qui oncques ses inexorables forces n'experimenterent? Il est bien vray que l'invisible ne se peult par chose visible proprement representer. Mais tout ainsi que par les choses crées $\&$ visibles [...] on peult veoir \& contempler l'inuisible Dieu \& incrée. Pareillement par les choses, esquelles la Mort a faict irreuocables passaiges, c'est ascauoir par les corps es sepulchres cadauerisez \& descharnez sus leurs monumentz, on peult extraire quelques simulachres de Mort (simulachres les dis ie vrayement, pour ce que simulachre vient de simuler, \& et faindre ce qui n'est point.) Et pourtant qu'on n'a peu trouuer chose plus approchante a la similitude de Mort, que la personne morte, on a d'icelle effigie simulachres, $\&$ faces de Mort, pour en noz pensees imprimer la memoire de Mort plus au vif, que ne pourroient toutes les rhetoriques descriptions des orateurs (Aiiir ${ }^{r}$.

Cette réflexion sur l'iconographie de la mort, caractérisée en tant que « curieux questionaire », n'est pas la première en Occident : Boccaccio, par exemple, avait déjà présenté des conceptions antiques de la mort dans un passage de sa De genealogia deorum $(\mathrm{I}, 32)$ qui était repris par le prédicateur Geiler de Kaysersberg dans le cycle de sermons tenu en $1495^{6}$. Elle est cependant parmi les premières à élaborer une approche presque phénomé-

4. La position érasmienne de Vauzelles et sa distance face à la Réforme sont soulignées par N. Z. DAvis, "Holbein's Pictures of Death and the Reformation in Lyon", Studies in the Renaissance, 3, 1956, pp. 97-130, part. pp. 113-118.

5. Voir H. GRABES, Mirror and Looking-Glass. Kontinuität und Originalität der Spiegelmetapher in den Buchtiteln des Mittelalters und der englischen Literatur des 13. bis 17. Jahrhunderts, Tübingen, 1973, « Anglia. Buchreihe, 16 » J. H. M. TAYLOR, « Un miroer salutaire », Dies illa. Death in the Middle Ages, id. éd., Liverpool, «Vinaver Studies in French, 1 », 1984, pp. 29-43; H. KIEPE, Die Nürnberger Priameldichtung. Untersuchungen zu Hans Rosenplüt und zum Schreib- und Druckwesen im 15. Jahrhundert, Munich, « Münchener Texte und Untersuchungen zur deutschen Literatur des Mittelalters, 74 », 1984, pp. 210, 246 ss.

6. La version latine parut pour la première fois en $1514 \mathrm{chez}$ Grüninger à Strasbourg (sous le titre Sermones prestantissimi), la version allemande, beaucoup plus courte dans la partie empruntée de la De genealogia deorum, chez le même en 1521 (Das buoch Arbore humana); V. Honemann, «Der Tod bei Geiler von Kaysersberg », Zeit, Tod und Ewigkeit in der Renaissance Literatur, James HoGg éd., vol. 1, Salzbourg, « Analecta Cartusiana, 117 », 1987, pp. 90107 ; Christian KIENING, Die schwierige Modernität. Der Ackermann des Johannes von Tepl im historischen Wandel, thèse d'habilitation, Munich, 1995, pp.161-166. Voir aussi les textes publiés par N. F. PAlmer, "Antiquitus depingebatur. The Roman Pictures of Death and Misfortune in the Ackermann aus Böhmen and Tkadlecek, and in the Writings of the English Classicizing Friars ", Deutsche Vierteljahrsschrift für Literaturwissenschaft und Geistesgeschichte, 57, 1983, pp. 171-239. 
nologique qui rejoint les résultats de la recherche moderne ${ }^{7}$. Vauzelles ne fait pas seulement mention du rapport entre la personnification de la Mort et la représentation funéraire de cadavres et squelettes (un rapport que les historiens d'art ont confirmé $)^{8}$, il souligne aussi la spécificité d'une représentation figurée ou allégorique de la mort : incapable de rendre visible un fait abstrait, elle pourrait quand même, en utilisant des éléments de la vie quotidienne, fonctionner comme un rapprochement. L'image de la Mort serait une image artificielle et approximative, une image appellative qui rend existant ce qui n'existe pas sur le plan visuel, afin d' « imprimer » aux humains «la memoire de Mort» (ibid.).

L'intention de cette explication est sans doute didactique, sinon rationalisante. Elle articule plutôt une théorie a posteriori qu'un programme iconographique. Elle tente à justifier les images bouleversantes de Holbein en les insérant dans le contexte d'un humanisme chrétien. Ceci ne fonctionne pas sans ambivalences. Aussi bien que Geiler de Kaysersberg, Vauzelles garde la tension entre les visualisations de la mort par des images et l'insistance sur l'impropre de ces visualisations. Dans la même phrase qui postule le caractère dissimulé de la figure de la Mort, il conçoit le corps mort en tant que résultat des « irreuocables passaiges » que la Mort aurait fait effectuer. Puis, expliquant le phénomène que les morts se « réaniment » dans les images et agissent «tresviuement ", tandis que les vivants apparaissent «tresmortement », il arrive à raconter une anecdote sur la fin du graveur Hans Lützelburger : «que la Mort craignant que ce excellent painctre ne la paignist tant vifue, qu'elle ne fut plus crainte pour Mort, \& que pour celà luy mesme n'en deuint immortel, que a ceste cause elle luy accelera si fort ses iours, qu'il ne peult paracheuer plusieurs aultres figures ià par luy trassées » (Aiiiv) ${ }^{9}$.

Malgré le ton évidemment ironique de ce passage maniériste qui crée l'image d'une Mort ambitieuse et vaniteuse, on aperçoit aussi la présence, même l'omniprésence de la figure de la Mort au début du $16^{\mathrm{e}}$ siècle. A ce moment les figurations s'étaient considérablement multipliées. Des cadavres et des squelettes peuplaient les textes et les images, apparaissaient sur les boîtes miniatures et médaillons, sur les boucles de ceinture, gaines de poignard et montres. La Mort vivifiée était partout. La multitude des apparitions fait comprendre pourquoi la recherche moderne s'est, d'une part, principalement contentée de classifier les images, de reconstituer leurs

7. L. de Vauzelles avait déjà publié, parmi les Blasons anatomiques du corps féminin, un Blason de la mort; voir L. DE VAuzelles, Poésies de Matthieu et de Jehan de Vauzelles, Lyon, 1877.

8. R. Helm, Skelett- und Todesdarstellungen bis zum Auftreten der Totentänze, Strasbourg, «Studien zur deutschen Kunstgeschichte, 255 », 1928; K. R. COHEN, Metamorphosis of a Death Symbol. The Changing Meaning of the Transi Tomb in the Late Middle Ages and the Renaissance, Berkeley-Los Angeles, 1974 ; K. BAUCH, Das mittelalterliche Grabbild. Figürliche Grabmäler des 11. bis 15. Jahrhunderts in Europa, Berlin-New York, 1976.

9. Lützelburger mourut en 1526 après avoir achevé 41 gravures sur bois ; E. His, « Hans Lützelburger, le graveur des Simulachres de la mort d'Holbein ", Gazette des Beaux-arts, 12, 4, 1870 , pp. $481-489$. 


\section{IMAGES DE LA MORT, DESTRUCTION DES IMAGES AU MOYEN AGE}

origines, d'examiner les traditions textuelles et iconographiques ${ }^{10}$ sans approfondir la connaissance des structures imaginatives ou des fonctions sociales $^{11}$; pourquoi, d'autre part, elle s'est laissée tenter de traiter les témoignages comme des sources sérielles ${ }^{12}$. Les grandes synthèses souffrent souvent d'une négligence face aux spécificités des témoignages, d'une précipitation poussive face aux rapports entre «imaginaire » et « réalité » sociale $^{13}$. L'explication la plus courante pour la reproduction extraordinaire des cadavres et des squelettes se réfère aux catastrophes épidémiques (comme la peste noire), par lesquelles le Bas Moyen Age était profondément ébranlé ${ }^{14}$. Des décès en masse auraient familiarisé les sociétés occidentales avec le phénomène paradoxal du cadavre, avec cette «synthèse négative entre identité et différence, proximité et distance, unité et séparation $\gg^{15}$. Néanmoins, les cas sont relativement rares où textes ou images réa-

10. Je me borne à quelques études principales. Pour le Moyen Age : T. Frimmel, Beiträge zu einer Ikonographie des Todes, Vienne, 1891 ; St. KozÁKy, A mulandóság ábrázolásai történeti fejlödésükben. Anfänge der Darstellungen des Vergänglichkeitsproblems, Budapest, « Bibliotheca humanitatis historica, 1 »1936; id., Danse macabre - Bevezetés : A haláltáncok elötti halál-didaktika. Danse macabre - Einleitung : Die Todesdidaktik der Vortotentanzzeit, Budapest, «Bibliotheca humanitatis historica, 5 », 1944 [en allemand avec des introductions en hongrois] ; L. E. Jordan (III), The Iconography of Death in Western Medieval Art to 1350, thèse, University of Notre Dame, 1980 ; Ann Arbor-Londres, 1980 (contient un répertoire iconographique jusqu'à 1350) ; V. PACE, «Dalla morte assente alla morte presente. Zur bildlichen Vergegenwärtigung des Todes im Mittelalter », Tod im Mittelalter, A. Borst éd. et al., Constance, «Konstanzer Bibliothek, 20 », 1993, pp. 335-376. Pour le $16^{\mathrm{e}}$ siècle : L. LuCHNER, Die Darstellung des Todes in der Deutschen Malerei bis zu H. Holbein d. J. (1550), thèse, Innsbruck, 1948 ; C. Martineau-GÉnieys, Le thème de la mort dans la poésie française de 1450 à 1550 , Paris, « Nouvelle Bibliothèque du Moyen Age, 6 », 1978 ; C. BLuM, La représentation de la mort dans la littérature française du XVI siècle, 2 vols, Paris, 1989.

11. Quelques exceptions : M. TitzmanN, «Der Tod als Figur im Drama des deutschsprachigen Gebiets im 16. Jhdt. : Implikationen und Transformationen ", Mélanges Alfred NoyerWeidner, K. W. HeMPfer et G. REGN éds, Wiesbaden, 1983, pp. 352-393 ; G. MATHIEUCASTEllani, Emblèmes de la mort. Le dialogue de l'image et du texte, Paris, 1988 ; P. DinZELBACHER, "Die tötende Gottheit : Pestbild und Todesikonographie als Ausdruck der Mentalität des Spätmittelalters und der Renaissance ", Zeit, Tod und Ewigkeit in der Renaissance Literatur, vol. 2, pp. 5-138.

12. La situation est différente pour les autels des pauvres âmes ou les ex-votos, où aussi des traditions de la longue durée peuvent être observées; voir G. et M. Vovelle, Vision de la mort et de l'au-delà en Provence d'après les autels des âmes du purgatoire, $X V^{e}-X X^{e}$ siècles, Paris, 1970.

13. J. SAUGNiEux, Les danses macabres de France et d'Espagne et leurs prolongements littéraires, Lyon, «Bibliothèque de la faculté des lettres de Lyon, 30 », 1972, commence le chapitre sur la signification de la danse macabre avec «les malheurs du temps». Le problème se pose également pour P. ARIÈs, L'homme devant la mort, Paris, "Univers historique ", 1977.

14. L'étude classique demeure celle de M. MeIss, Painting in Florence and Siena after the Black Death. The Arts, Religion, and Society in the Mid-Fourteenth Century, Princeton, 1951 ; en outre J. POLzER, « Aspects of the Fourteenth-Century Iconography of Death and the Plague », The Black Death, D. Williman éd., New York, 1977, pp. 108-130; R. E. LeRner, « The Black Death and the Western European Eschatological Mentalities", The American Historical Review, 86, 1981, pp. 533-552.

15. T. H. MACHO, Todesmetaphern. Zur Logik der Grenzerfahrung, Francfort, 1987 (Édition Suhrkamp, 1419), p. 410 ss. ( Negative Synthesis zwischen Identität und Differenz, Nähe und Entfernung, Einheit und Trennung, etwas und nichts »); cf. aussi L.-V. ThOMAs, Le cadavre. De la biologie à l'anthropologie, Paris, 1980 ; J.-T. MAERTENS, Le jeu du mort. Essai d'anthropologie des inscriptions du cadavre, Paris, 1979. 
gissent directement à des événements historiques. Même l'exemple jadis le plus évident, le Trionfo della Morte de Pise, s'est dévoilé en tant que témoignage "précoce» (1330-1336) de la grande catastrophe de $1348^{16}$. Pour esquiver le problème de la référence historique (directe), on a utilisé l'expression de « sensibilité macabre » qui, nourrie d'une atmosphère chiliastique, aurait établi un regard spécifique, oscillant entre l'horreur et la délectation, sur la décomposition du corps humain ${ }^{17}$. Mais là aussi, la question se pose si ce terme aide vraiment pour comprendre la «réanimation » des cadavres, des morts à la fin du Moyen Age. Ne cache-t-il pas l'entremêlement de conceptions, imaginations et figures? Ne superpose-t-il pas les aspects se référant à l'acte de mourir et les abstractions se rapportant au phénomène de la mort ? Si on constate « qu'il y avait [...] aucune différence essentielle entre la représentation du cadavre [...], la représentation de l'inexorable traîtrise de la Mort [...] et les images des fantômes qui constituent la mise en scène de la Danse macabre ${ }^{18}$, n'est-on pas quand même obligé de prendre en considération ce manque de différence au lieu de l'inscrire dans une mentalité plus ou moins vague ?

Le problème consiste dans le fait qu'on a affaire à des phénomènes qui, bien que liés, se situent sur des plans logiques différents : la représentation de vrais «autres », les morts, et la représentation de l'«autre » du Soi, la mort. La mort, d'un côté, conçue en tant que limite ou en tant que transitus, représente un abstractum, une connaissance latente, le joint toujours insaisissable entre ce qui est pensable et ce qui est impensable. Les morts, de l'autre côté, représentent une réalité ; ils subsistent paradoxalement dans la forme de leurs corps morts et, aussi, par la mémoire des vivants, mémoire qui peut les «réanimer» de nouveau ${ }^{19}$. C'est dans la figure du cadavre ou du squelette que les deux notions se rejoignent et qu'elles se mêlent d'une façon difficile à saisir : à savoir dans toutes les nuances entre le cadavre parlant, les morts agissants, la figure démonstrative et l'être presque démoniaque tel qu'il apparaît dans les scénarios du jugement dernier ou du Trionfo della Morte. Le spectre des nuances démontre aussi que les textes et surtout les images ne se laissent pas complètement insérer dans une idéologie cléricale qui tentait de gouverner l'expérience de la mort pour toute une société. En fait, les témoignages gardent à l'intérieur un moment anarchique et subversif - à travers tous les apprivoisements. Michel Vovelle, indiquant que la figure squelettique de la Mort porterait déjà le caractère d'une « conceptualisation » (ce qui se voit clairement dans le processus d'amaigrissement des

16. L. Bellosi, Buffalmacco e il Trionfo della Morte, Turin, 1974. Toute une série de travaux sur le Camposanto de Pise seront prochainement publiés, par exemple: Friederike Wille, thèse, Munich.

17. Voir déjà le chapitre très expressif et influent de J. Huizinga sur l'image de la Mort (Herfstij der middeleeuwen, 1919, $5^{\text {e }}$ éd. 1941 ; trad. allemande, pp. 190-208).

18. A. TENENTI, Il senso della morte e l'amore della vita nel Rinascimento (Francia e Italia), Turin, 1957 ; trad. frse : Sens de la mort et amour de la vie. Renaissance en Italie et en France, Paris, L'Harmattan, 1983, v. commentaire des illustrations 14-16.

19. Voir J.-C. SCHMIT, Les revenants. Les vivants et les morts dans la société médiévale, Paris, 1994. 


\section{IMAGES DE LA MORT, DESTRUCTION DES IMAGES AU MOYEN AGE}

cadavres jusqu'au squelette $)^{20}$, souligne en même temps que les morts anarchiques et agressifs n'étaient pas expulsés de l'imagination du monde populaire et que peut-être là " se fait l'une des césures essentielles entre culture populaire et culture d'élite, religion populaire et religion d'élite ${ }^{21}$.

Néanmoins, c'est justement dans les œuvres complexes des artistes savants, comme Holbein, que les tensions entre l'abstrait et le concret, entre les normes théologiques et les transgressions anarchiques semblent le mieux conservées. Également dans l'épître dédicatoire des Simulachres, quoique Vauzelles fasse dériver la figure de la Mort des représentations funéraires des morts, ce sont ces derniers qu'il voit agir « tresuiuement ». Ceci indique que l'image de la Mort se fait comprendre en tant qu' « image stratifiée $»^{22}$ par excellence. Si l'on accepte que les représentations de la mort/Mort n'expriment pas seulement des variations sur le thème constant du memento mori, on découvre des rapports complexes entre idées et formes, textes et images, didactique et esthétique, on découvre l'implication du spectateur / lecteur dans les œuvres et « le jeu de miroir entre les vifs et les morts $»^{23}$. Le jeu d'une ressemblance dissemblable, d'une inégalité temporelle, d'un déplacement, jeu qui fait apparaître une autre figure que celle de la mort : celle du double, alternant entre le Soi et l'« autre », tel qu'il était reconstitué pour quelques figures de la Grèce ancienne par JeanPierre Vernant ${ }^{24}$ et tel qu'il était défini par Edgar Morin : « universellement, la décomposition est la période terrible où corps et double sont encore mêlés l'un à l'autre, où tout n'est pas accompli, où plane une sourde menace vampirique. [...] Le macabre médiéval du $15^{\mathrm{e}}$ siècle et le macabre espagnol, avec leurs morts décharnés, grinçants, horribles, squelettes recouverts ou non de lambeaux de chair, sont des ossements-cadavres possédés par le double. Là où la conscience des vivants n'arrive plus à dissocier nettement le double du cadavre, là où le double demeure plus ou moins englué au cadavre, là règne la terreur ${ }^{25}$.

Or, ce double dans son caractère décharné ou squelettique, auquel les observations suivantes sont consacrées, est moins une figure mythique qu'une figure artificielle et expérimentale. Bien que les témoignages qui seront discutés semblent parfois proches des conceptions traditionnelles (dites «primitives ») d'un double à la fois matériel et spirituel, documenté par l'ethnologie à travers les cultures, il faut renoncer à tirer des parallèles

20. Voir aussi P. CARus, «The Skeleton as a Representation of Death and the Dead», The Open Court, 22, 1908, pp. 620-635 ; W. ECKART, « Die Darstellung des Skeletts als Todessymbol in der Sinnbildkunst des 16. und 17. Jahrhunderts (1) », Studien zur Thematik des Todes im 16. Jahrhundert, Paul Richard BLuM éd., Wolfenbüttel, "Wolfenbütteler Forschungen, 22 », 1983, pp. 21-47.

21. M. Vovelle, La mort en Occident de 1300 à nos jours, Paris, 1983, p. 125 ; exemples de la popularisation d'un motif, celui de la Mort chevauchant un bœuf (cf. le comte A. DE LABORDE, La Mort chevauchant un bouf. Origine de l'office des morts dans certains livres d'heures de la fin du XVe siècle, Paris, 1923), chez A. B. Rooth, Döden och den svarta oxen, Uppsala, 1985.

22. J. ZiEgler, Les vivants et la mort, Paris, 1975, p. 27.

23. J. WIRTH, La jeune fille et la mort. Recherches sur les thèmes macabres dans l'art germanique de la Renaissance, Genève, « Hautes Études médiévales et modernes, 36 », 1979, p. 19. Des analyses convaincantes se trouvent aussi chez F. BÄCHTIGER, Vanitas - Schicksalsdeutung in der deutschen Renaissancegraphik, thèse, Munich, 1970.

24. J.-P. VeRnANT, L'individu, la mort, l'amour. Soi-même et l'autre en Grèce ancienne, Paris, «Bibliothèque des Histoires », 1989.

25. E. Morin, L’homme et la mort, 3éd., Paris, 1976, p. 157. 
séduisants, mais superficiels. Ils ne pourraient pas rendre compte de la constellation historique dans laquelle les textes et les images apparaissent et du plan conceptionnel des œuvres par lequel les attitudes se définissent. Sans doute les œuvres transportent-elles des éléments d'un « complexe anthropologique fondamental $»^{26}$, mais en même temps elles font partie d'un univers où le mythe n'est réintroduit qu'en second ordre, où la didactique cléricale s'interpénètre avec des imaginations populaires, où les fantasmes relatifs aux rencontres de vivants et de morts sont imprégnés d'une psychologie subtile, où les « autres » se manifestent comme figures du Soi-même et dévoilent le «propre» de l'existence humaine. Pour aborder le caractère de ces «doubles décomposés » je choisis un point qui, bien qu'étant déjà un point final, semble le mieux s'adapter à la description d'Edgar Morin. Je commence avec Pierre Bruegel l'Ancien dans les années 1562-1563, avec un tableau qui, dans sa condensation extraordinaire, n'est point représentatif pour une mentalité collective mais qui assimile un nombre considérable de traditions, ce qui permet de définir les enjeux essentiels.

\section{Les enjeux}

Triumph van den Doot, c'est le titre probablement authentique qui est donné au tableau (aujourd'hui au Prado, Madrid) dans l'inventaire d'héritage de Philips van Valkenisse (1614) ${ }^{27}$. Triomphe de la Mort, ceci se réfère d'abord sans doute à la figure à cheval et avec la faux, qui est placée justement au centre du tableau ; une figure qui correspond à un type iconographique, courant particulièrement en Italie dès le $14^{\mathrm{e}}$ siècle ${ }^{28}$. Il se peut que Bruegel ait vu des représentations semblables pendant un voyage dans les pays méridionaux qu'il aurait effectué au cours des années 1552-155329. Mais il a profondément transformé le thème. La figure isolée de la Mort que les illustrations des manuscrits et les peintures murales italiennes présentent, soit volante soit en char ou à cheval, est réintégrée chez Bruegel dans une masse de morts qui attaquent les vivants. La figure ne se détache principalement que par sa position dans la composition, par sa place au point d'inter-

26. Un mélange de cultures et d'époques se trouve chez C. Lecouteux, Fées, sorcières et loups-garous au Moyen Age. Histoire du double, Paris, 1992 (cité p. 171); R. BOYER, Le monde du double. La magie chez les anciens Scandinaves, Paris, 1986. Méthodiquement plus nuancé C. Ginzburg, Storia notturna. Una decifrazioni del sabba, Turin, « Biblioteca di cultura storica, $176 », 1989$.

27. Voir Bruegel l'Ancien. Introduction par C. DE TOLNAY. Documentation par P. BIANCONI, Paris, 1968, 2e éd. "Les classiques de l'art », 1988, p. 98. Pour une bibliographie voir R. H. MARIJnissen, Bruegel : tout l'ouvre peint et dessiné, Anvers-Paris, 1988 (éd. néerlandaise, p. $195 \mathrm{ss})$.

28. Voir Prince d'Essling et E. Müntz, Pétrarque, les études d'art, son influence sur les artistes, Paris, 1902; L. GUERRY, Le thème du triomphe de la mort dans la peinture italienne, Paris, 1950 ; A. Tenenti, La vie et la mort à travers l'art du $X V^{e}$ siècle, Paris, 1952, $2^{\mathrm{e}}$ éd. «Cahiers des Annales, 8 », 1983, pp. 17-27. Sur des racines possibles de la Mort ailée dans l'art byzantin ou étrurien, A. Reuter, Beiträge zu einer Ikonographie des Todes, Leipzig, 1913; I. KRAUSKOPF, Todesdämon und Totengötter im vorhellenistischen Etrurien, Florence, 1987.

29. On a pensé notamment au Trionfo de Palerme ; F. Grossmann, Bruegel: The Paintings, Londres, 1955 ; M. G. PAOLINI, "Il Trionfo della Morte di Palermo e la Cultura Internazionale ", Rivista dell'Istituto Nazionale d'Archelogia e Storia dell'Arte, 20, 1963, pp. 301-369. 


\section{IMAGES DE LA MORT, DESTRUCTION DES IMAGES AU MOYEN AGE}

section de deux espaces bien distingués dans l'image. Dans un premier plan scénique se rangent des groupes de figures plus ou moins isolés, figures squelettiques, fonctionnant ici en tant que personnifications de la Mort, et figures humaines représentant des positions différentes dans la société : l'empereur, auquel la Mort montre l'horloge du sable; le cardinal, épaulé par la Mort ; un pèlerin égorgé par un autre squelette en cotte de maille. Des types empruntés des danses macabres rejoignent ici d'autres types iconographiques déjà connus : la Mort et le couple amoureux, la Mort comme fossoyeur, comme meurtrier, comme lansquenet concupiscent ${ }^{30}$.

Ce qui s'enfonce dans ce premier plan, ce sont les armées de morts, venant de l'arrière-plan, les morts de la mer qui «tempêtent » sur les eaux, les morts de la terre qui se lèvent de tombes et d'ossuaires, qui submergent les collines, qui anéantissent tout ce qui croise leur chemin. Les vivants sont pourchassés comme des animaux, quelques-uns piégés au filet, d'autres noyés ou roués, exécutés par l'épée ou la corde. Bruegel combine des aspects métaphoriques et concrets ${ }^{31}$, images du trépas et visions de la mortalité, il rend palpable tout ce qui peut s'associer à la mort dans l'agissement des squelettes. Le temps aussi devient signe de la mort : un squelette utilise ses bras comme aiguilles de la montre. Une usurpation complète du pouvoir par les morts qui tuent les vivants, enlèvent et enterrent les cadavres, sonnent les glas funèbres. Une usurpation de la masse qui illustre en même temps la victoire absolue de la Mort/mort.

L'ambivalence du triomphe de la Mort et du triomphe des morts-vivants semble donner la clef pour la compréhension du tableau. Les morts/Morts apparaissent plus que vivants, ils assument les fonctions des vivants, même celle de la réflexion - ce qu'on voit tout à gauche par la figure d'un squelette pensif, presque mélancolique. Bruegel ne s'arrête pas à l'illustration du contraste extrême entre les vifs et les morts, il développe un rapport de miroitement. Il conçoit les morts en tant que doubles surpuissants qui se travestissent et enfilent des masques, qui jouent les rôles des vivants pour les effrayer et les remplacer. Avec un mélange de curiosité et d'incompréhension un homme parmi ceux qui sont piégés au filet regarde la Mort sous son froc (fig. 1). Et c'est ce moment-là que le tableau fixe : le moment d'une incompréhension qui fait toucher à la folie, le moment de l'ébahissement et de la stupeur du côté des vivants. Bruegel fixe le cri strident de ceux qui ne veulent pas mourir, qui - à la différence des vivants des danses macabres du $15^{\mathrm{e}}$ siècle - se cabrent désespérément contre une mort qui n'est plus évidente et n'est même plus souhaitée par les infirmes et les malades. Une mort sans espoir, non plus apprivoisée par un modèle du salut. Les hommes tentent de lui résister dans un combat inégal (un chevalier en rouge en train de tirer son épée) ou de la fuir (un bouffon en train de se cacher sous la table ; fig. 1). Pour les vivants du tableau, les morts demeurent les « autres », les étrangers, les horribles. Mais le spectateur voit plus : il voit aussi la trans-

30. Voir F. BÄCHTIGER, op. cit., pp. 88-150 (« Vanitas und Liebespaar »; «Tod und Landsknecht »); H. Theissing, Dürers Ritter, Tod und Teufel. Sinnbild und Bildsinn, Berlin, 1978.

31. Voir aussi A. Dundes et C. A. StIBBe, The Art of mixing Metaphors : A Folkloristic Interpretation of the "Netherlandish Proverbs » by Pieter Bruegel the Elder, Helsinki, «FF communications, $230 », 1981$. 


\section{KIENING}

RENCONTRES DES VIVANTS ET DES MORTS
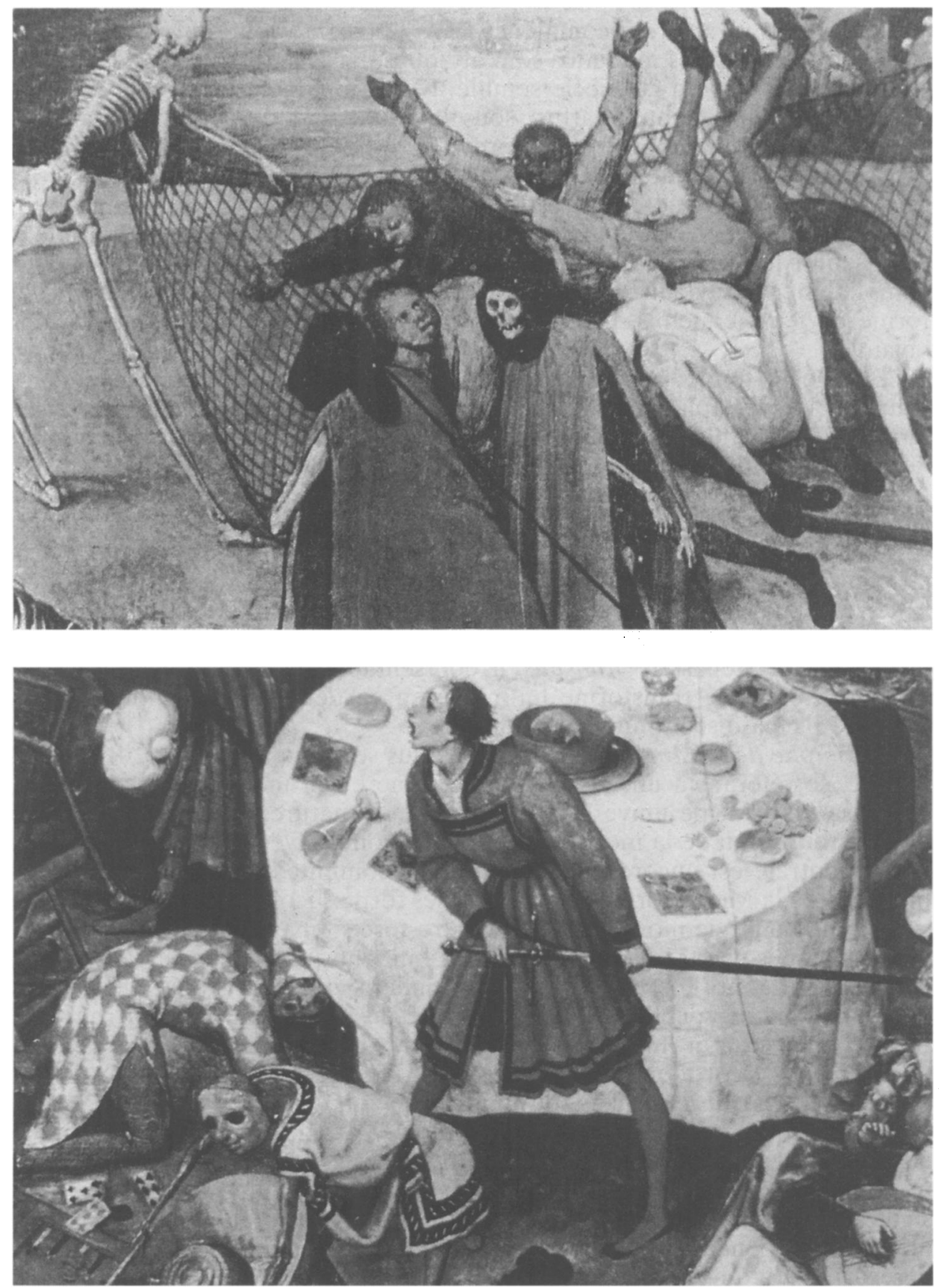

Fig. 1. - Bruegel, Le Triomphe de la Mort, vers 1562, huile sur panneau, détail (Madrid, Prado; photographies de l'auteur).

En haut, la Mort piège les vivants au filet.

En bas, la lutte contre la Mort. 


\section{IMAGES DE LA MORT, DESTRUCTION DES IMAGES AU MOYEN AGE}

formation des vivants qui commence dans la partie droite du premier plan. Tandis qu'ailleurs des individus sont attaqués par les morts, il s'y déroule un « holocauste » qui au $20^{\mathrm{e}}$ siècle semble avoir un caractère presque prophétique : les vivants sont rabattus, sous le roulement du tambour, dans un coffre noir, une "immense souricière ", qu'on peut comprendre comme un espace de transformation. Car les nouvelles armées de morts qui apparaissent dans la partie tout à droite du tableau, qui sont déjà munies de lances et attendent évidemment le signal pour leur intervention, semblent représenter justement ceux qui étaient rabattus dans le coffre. Décomposés complètement, devenus squelettes, les morts n'ont pas seulement pris la forme de leurs ennemis, mais, eux aussi, ils sont devenus les ennemis des vivants.

Bruegel a fondé la composition picturale sur un double mouvement : celui de l'arrière-plan vers l'avant-plan qui détache, parmi la masse des morts indiscernables, quelques-uns se rapprochant de la personnification de la Mort ; et celui, au milieu du tableau, de gauche à droite, qui déclenche la transformation des vivants en morts. Au point d'intersection des deux mouvements se situe la figure «triomphante » de la Mort à cheval. Ainsi, Bruegel joue sciemment avec la tension de notions différentes : l'image des morts en tant que masse anonyme, en tant que cadavres surgissant de la mer et de la terre ; l'image de la Mort en tant que figure personnifiée ; la notion de la mort en tant qu'événement, en tant qu'acte de mourir. Comme cependant le processus du trépas, le transitus irreprésentable est placé en dehors du tableau, celui-ci se transforme lui-même en partie d'un espace plus grand, défiguré par la discontinuité temporelle.

Triomphe de la Mort, cela ne signifie plus seulement un thème iconographique, se référant à une figure démoniaque et en même temps abstraite, cela vise un principe universel : le monde et la nature, le temps et l'espace dans le tourbillon de la mort. Ainsi Bruegel rompt avec le modèle auquel la figure centrale est encore due. Car il ne se contente pas d'illustrer l'omniprésence de la Mort/mort à travers diverses formes iconographiques, il libère surtout une énergie mortelle, une « contre-force » inapprivoisable, il pousse l'agression des « autres " vers un point culminant qui exclut une consolation chrétienne. La mort est partout, l'espoir nulle part. La résistance des vivants demeure sans perspective. Derrière la tête du noble chevalier en rouge se trouve, inaperçu par lui-même, un crâne dans une coupe. Lui aussi, personnification du cri strident des vivants, n'est éloigné que par quelques centimètres, que par un déplacement minimal de son destin (fig. 1).

Ce motif du crâne dans une coupe mérite l'attention parce qu'il renvoie au thème du fruit mortel, du péché originel en tant que point initial de la mortalité humaine. Mais là aussi, Bruegel a changé l'élément chrétien en un signe de la transformation qui a saisi la nature en sa totalité. Il a isolé l'élément de son pendant : c'est-à-dire du triomphe sur la mort par la passion et la résurrection du Christ. Même si les squelettes attaquent les vivants sous le signe de la croix, Bruegel ne reprend pas le thème de la bonne mort chrétienne, mais plutôt le thème contemporain des guerres de Religion - telles que celle entre catholiques et huguenots, commencée en 1562, l'année probable du commencement du Triomphe de la Mort. Ce sont les horreurs de la 
guerre que Bruegel fait apparaître, horreurs d'une guerre devenue incontrôlable et uniformisante. Dans ce contexte, la mort n'a plus de correctif chrétien, n'est plus concevable en tant que transitus pour le salut ou la damnation, mais en tant que transitus paradoxal intra-mondain, causé par ceux qui s'élèvent, d'une façon pas du tout chrétienne, de leurs tombes. Le scénario de cette "résurrection » est apocalyptique, l'horizon embrasé, le monde touché par l'enfer. D'un donjon au centre du tableau jaillissent des êtres monstrueux inspirés sans doute par Jérôme Bosch. Une apocalypse paradoxale, sans dieu, sans au-delà, qui laisse ouvert s'il s'agit d'un déclin ou d'une inversion du monde.

Face à cette composition obstinée et complexe, la question se pose si le Triomphe de la Mort peut revendiquer une quelconque représentativité ou s'il ne témoigne «de la personnalité irréductible » du peintre, «d'expressions atypiques ou inclassables ${ }^{32}$. Sans doute, le tableau se laisse insérer dans le contexte de l'œuvre de Bruegel et de la peinture néerlandaise du $16^{\mathrm{e}}$ siècle $^{33}$; sans doute, sa conception se fait mieux comprendre si on regarde les relations de Bruegel avec quelques savants libertins des Pays$\mathrm{Bas}^{34}$. Ce qui y importe plus ce sont les tensions entre les traditions iconographiques que Bruegel a reprises et qu'il a transformées en un jeu inquiétant avec les miroitements et déplacements qui s'effectuent dans les rencontres des vivants et des morts. Les « doubles décomposés » tels qu'ils apparaissent dans le Triomphe de la Mort ou dans les Simulachres de Holbein ont acquis une liberté de mouvement qui surpasse beaucoup celle des vivants. Ils ne sont plus des figures, mises en scène pour rappeler la caducité de la vie humaine et l'inexorabilité de la mort, ils exposent plutôt la difficulté de la conscience humaine de penser la mort en tant que abstractum. Ils ne renvoient plus à une assimilation de la mort à l'aide de l'appareil chrétien de l'art de mourir, mais représentent plutôt une tentative de cerner les limites de l'expérience humaine. Les images de Bruegel et Holbein, extrêmes dans leur radicalité, forment ainsi le point culminant d'un processus, dont les origines remontent au moins au $12^{\mathrm{e}}$ ou $13^{\mathrm{e}}$ siècle.

\section{Premières rencontres}

Aux $12^{\mathrm{e}}$ et $13^{\mathrm{e}}$ siècles, on trouve les premiers textes du Moyen Age (pour la plupart en latin) où la Mort apparaît comme interlocuteur ${ }^{35}$. Souvent, ses traits «physiques » sont encore peu développés. Dans les Versus de morte et

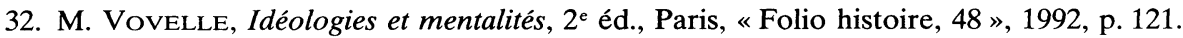

33. Voir H. Dollmayr, «H. Bosch und die Darstellung der vier letzten Dinge in der niederländischen Malerei des 15. und 16. Jahrhunderts ", Jahrbuch der Akademie der Künste, 19, 1898, pp. 284-343.

34. Bruegel l'Ancien, op. cit., p. 86.

35. Les textes sont traités en dernier lieu par J. RoTH, The Ackermann aus Böhmen and the Medieval "Streitgespräch », thèse, Chicago, Ill., 1981, et (d'une manière peu satisfaisante) par G. E. K. THIEL, Die Todesfigur : Eine Studie ihrer Funktion in der deutschen Literatur vom vierzehnten bis zum sechzehnten Jahrhundert unter besonderer Beruecksichtigung des sozial- und gesellschaftskritischen Aspekts, thèse Univ. of Natal, Durban, 1989 ; voir aussi C. KIENING, op. cit., pp. 127-195. 


\section{IMAGES DE LA MORT, DESTRUCTION DES IMAGES AU MOYEN AGE}

divite par exemple, conservés dans un seul manuscrit du milieu du $12^{\mathrm{e}}$ siècle, écrit par le moine Louis en Hainaut, la Mort barre le chemin au riche, rappelle la nécessité d'une vie plus spirituelle et annonce la mort du riche pour la nuit suivante ; sa figure cependant demeure imprécise et n'est pas directement liée à la description de la décomposition du corps humain (v. 73-102). En revanche, dans le Dialogus mortis cum homine, créé éventuellement aussi au $12^{\mathrm{e}}$ siècle en Allemagne du Sud, très répandu jusqu'à la fin du $15^{\mathrm{e}}$ siècle et traduit ou adapté dans plusieurs langues vernaculaires, la Mort prend pour la première fois explicitement l'aspect d'un cadavre. Venant de la région du Styx, elle se présente par son annonce Eritis consimiles $(6,4)$ en tant que double décomposé, décalé dans le temps, et en même temps, avec son instrument, la faucille, en tant que force violente. Ainsi, des catégories métonymiques et métaphoriques interfèrent ${ }^{36}$. Les définitions que la Mort déploie en réponse aux questions de l'homme offrent toute une série d'aspects fonctionnels ; ils esquissent l'image d'un juge supratemporel, d'un souverain des enfers, d'un signe visuel pour l'avenir de tous les humains, d'une contre-force qui (à travers les vers) retire les humores. La Mort est figura et signum en même temps : elle est incarnation de la force abstraite par laquelle la vie humaine est déterminée et représentation du processus biologique concret qui transpose la vie en non-vie.

Le caractère expérimental de cette figure cadavéreuse de la Mort, qui a stimulé la production d'illustrations impressionnantes au $15^{\mathrm{e}}$ siècle (fig. 2 ), se révèle clairement en comparaison avec les figures semblables de la fin du Moyen $\mathrm{Age}^{37}$. La Mort du Dialogus ne prononce pas l'exhortation stéréotypée à la dévotion et ne s'exprime pas en termes sotériologiques ou eschatologiques, son discours se concentre plutôt sur le sort physique de l'homme et provoque un mélange d'horreur et de curiosité qui est également mis en scène, comme on l'a vu, dans les réactions des quelques individus dans le Triomphe de la Mort de Bruegel. Ainsi, le Dialogus rejoint un intérêt croissant à l'apparence des morts et aux aspects de la décomposition du corps humain qui se manifeste dès le $12^{\mathrm{e}}$ siècle ${ }^{38}$. L'un des énoncés les plus évidents est celui de Guillaume de Newburg qui, dans son Historia rerum anglicarum, s'étonne «que les cadavres des morts sortant de leur sépulture sont poussés à errer par je ne sais quel esprit, soit pour terrifier les vivants, soit pour leur nuire, et qu'ils retournent à ces mêmes tombeaux qui s'ouvrent

36. M. TitzMAnN, op. cit., p. 371 , souligne (à propos du drame du $16^{\mathrm{e}}$ siècle) que la figure de la Mort «vereinigt in sich beide Terme einer Metonymie : zeichenhaft repräsentiert sein Attribut die (unsichtbare) Ursache (das Tötende), seine Gestalt die (sichtbare) Wirkung (das Getötete). Er ist, wozu jedermann wird (das Ergebnis) und wodurch jedermann es wird (das Ereignis). Er bringt jedermann um seine Gegenwart und er repräsentiert zugleich jedermanns Zukunft, der der Held in ihm begegnet ».

37. Sur la reception du Dialogus voir C. KIENING, p. 129 ss, p. 159 ss.

38. A propos de Heinrich von Melk et Hélinand de Froidmont G. Scholz Williams, The Vision of Death : A Study of the Memento mori Expressions in some Latin, German, and French Didactic Texts of the 11th and 12th Centuries, Göppingen, "Göppinger Arbeiten zur Germanistik, 191 », 1976 ; sur les Débats de l'âme et du corps (Visio Philiberti) T. BATIOUCHKOFF, «Le débat de l'âme et du corps », Romania, 20, 1891, pp. 1-55 et 513-578. 
d'eux-mêmes ${ }^{39}$. Cependant, les morts dont les auteurs (surtout des clercs) imaginaient l'apparition n'y sont pas des doubles et ne surgissent pour la plupart pas directement des tombeaux. Il s'agit principalement des personnes «historiques» avec lesquelles le visionnaire/rêveur est familier, souvent des parents ; leur apparition est due au «contrat » entre les générations, à la réciprocité entre l'ici-bas et l'au-delà, aux soucis et craintes des vivants face aux morts ${ }^{40}$. Par contre, les morts qui, comme le dit Guillaume de Newburg, «sortent de leurs tombeaux » pour terrifier les vivants par leur simple apparition sont presque sans exception anonymes, ce qui les qualifie de doubles aussi bien que de personnifications de la mort.

Un des types les plus influents est la Légende ou le Dit des Trois Vifs et des Trois Morts qui était assez répandu du $13^{\mathrm{e}}$ jusqu'au $16^{\mathrm{e}}$ siècle avec de nombreuses variantes et qui se reflète éventuellement encore chez Bruegel dans le groupe des trois squelettes avec leurs cercueils, sur le bord droit du tableau ${ }^{41}$. La Légende (qui est plutôt un exemplum qu'une véritable légende) met en scène trois hommes (par exemple des rois ou des nobles) rencontrant trois morts qui sont parfois debout, parfois couchés dans leurs sépultures, parfois aussi en train de s'en relever, trois morts qui démontrent à leurs pendants la vanité de la vie terrestre. La disposition symétrique des vifs et des morts est soulignée par des attributs similaires (fig. 2), ou parfois par une gradation des âges de la vie d'une part, des degrés de décomposition de l'autre. De même, la phrase centrale de la Légende: Quod fuimus estis, quod sumus eritis ( $\mathrm{Ce}$ que vous êtes, nous l'étions. Ce que nous sommes, vous deviendrez »), qui se trouve déjà en arrière-plan du Dialogus mortis cum homine et qui est souvent cité ailleurs, rappelle l'identité sous-jacente des morts et des vifs qui ne se distinguent que par le temps écoulé.

Le but de la Légende est une prise de conscience des vivants de leur propre vie et de leur propre avenir. Au modèle de la conversio effectuée par la confrontation avec la vanité - modèle qui existe aussi dans le monde oriental et arabe dans lequel ont été cherchées les origines de la Légende elle a donné un aspect véritablement chrétien : la scène est souvent intégrée dans une vision, située sous une croix et commentée par un ermite - ce qui permettait de souligner le caractère autant extraordinaire qu'exemplaire de l'événement, mais ce qui peut aussi se comprendre comme le souci de «christianiser» un thème ambigu ${ }^{42}$. En fait, le développement historique de

39. Guillaume DE NewBURg, Historia rerum anglicarum usque ad annum 1198, R. HowLETT éd., Chronicles of the Reigns of Stephen, Henry II and Richard I, vol. I, Londres, « Rolls Series ", 1884, p. 477 ; traduction dans C. LeCOUTEUX et P. MARCQ, Les esprits et les morts, Paris, 1990, p. 171 (citée) ; et (légèrement différente) dans J.-C. SCHMITT, op. cit., p. 80.

40. J. C. SchmitT, op. cit., ; outre la littérature citée par J.-C. SCHMITT voir aussi St. CosACCHI, Makabertanz. Der Totentanz in Kunst, Poesie, Brauchtum des Mittelalters, Meisenheim/ Glan, 1965, pp. 285-396.

41. Les études principales sont: K. Künstle, Die Legende der drei Lebenden und der drei Toten und der Totentanz, Fribourg, 1908 ; S. Glixelli, Les cinq poèmes des trois morts et des trois vifs, Paris, 1914 ; W. Rotzler, Die Begegnung der drei Lebenden und der drei Toten. Ein Beitrag zur Forschung über die mittelalterlichen Vergänglichkeitsdarstellungen, Winterthur, 1961 ; C. SetTis-Frugoni, «Il tema dell'incontro dei trè vivi e dei trè morti nella tradizione medievale », Atti della Accademia Nazionale dei Lincei 364, Rome, 1967, pp. 145-252.

42. M. Vovelle, La mort en Occident, op. cit., p. 113. 
IMAGES DE LA MORT, DESTRUCTION DES IMAGES AU MOYEN AGE

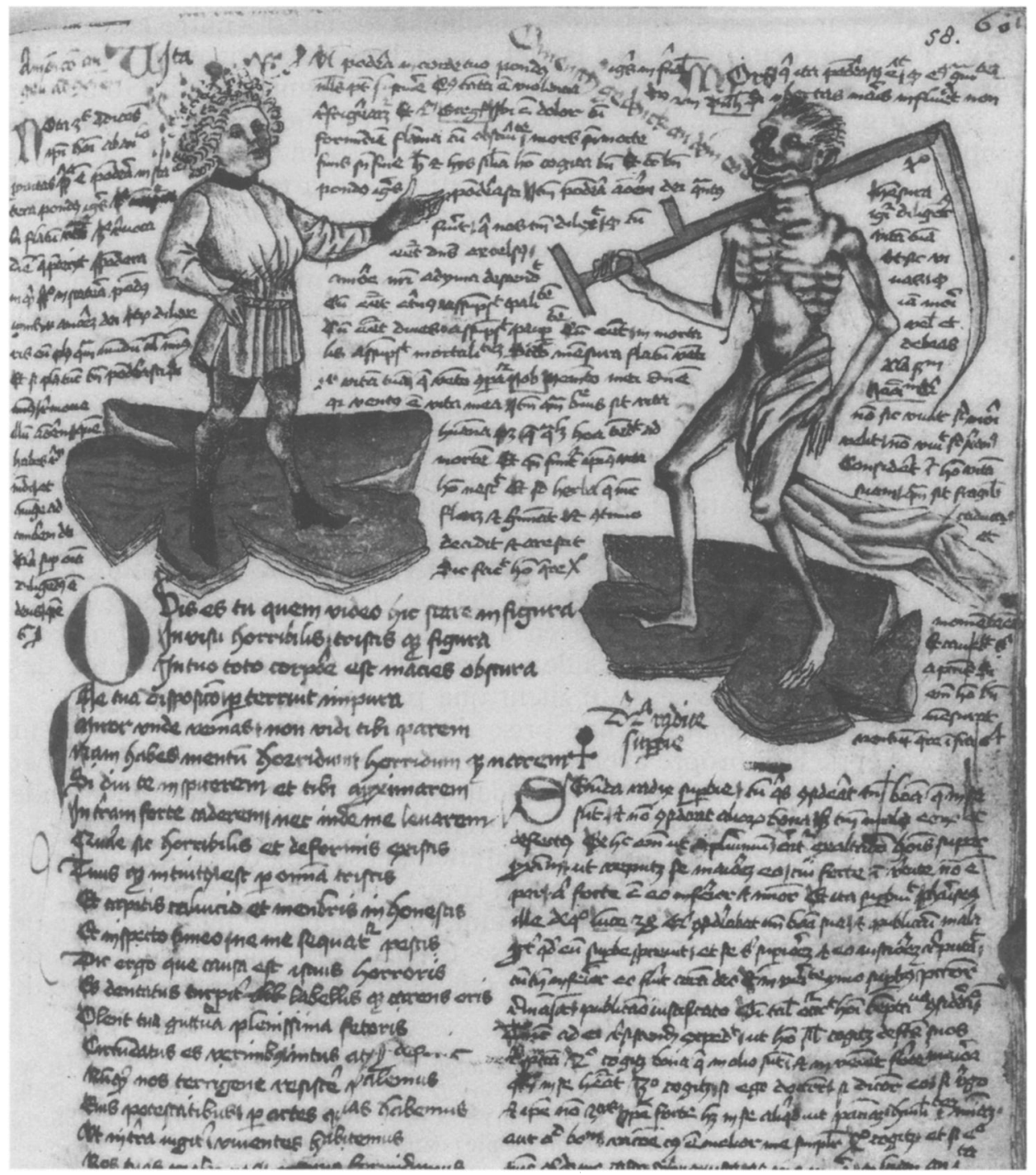

Fig. 2. - Dessins à la plume, colorés (Munich, Bayerïsche Staatsbibliothek, cgm 3974 , vers 1460 ; photographies de la bibliothèque).

Ci-dessus, Dialogus mortis cum homine (fol. $58^{\mathrm{r}}$ ).

Ci-contre, Rencontre de trois rois morts et de trois rois vifs (fol. 59r). 


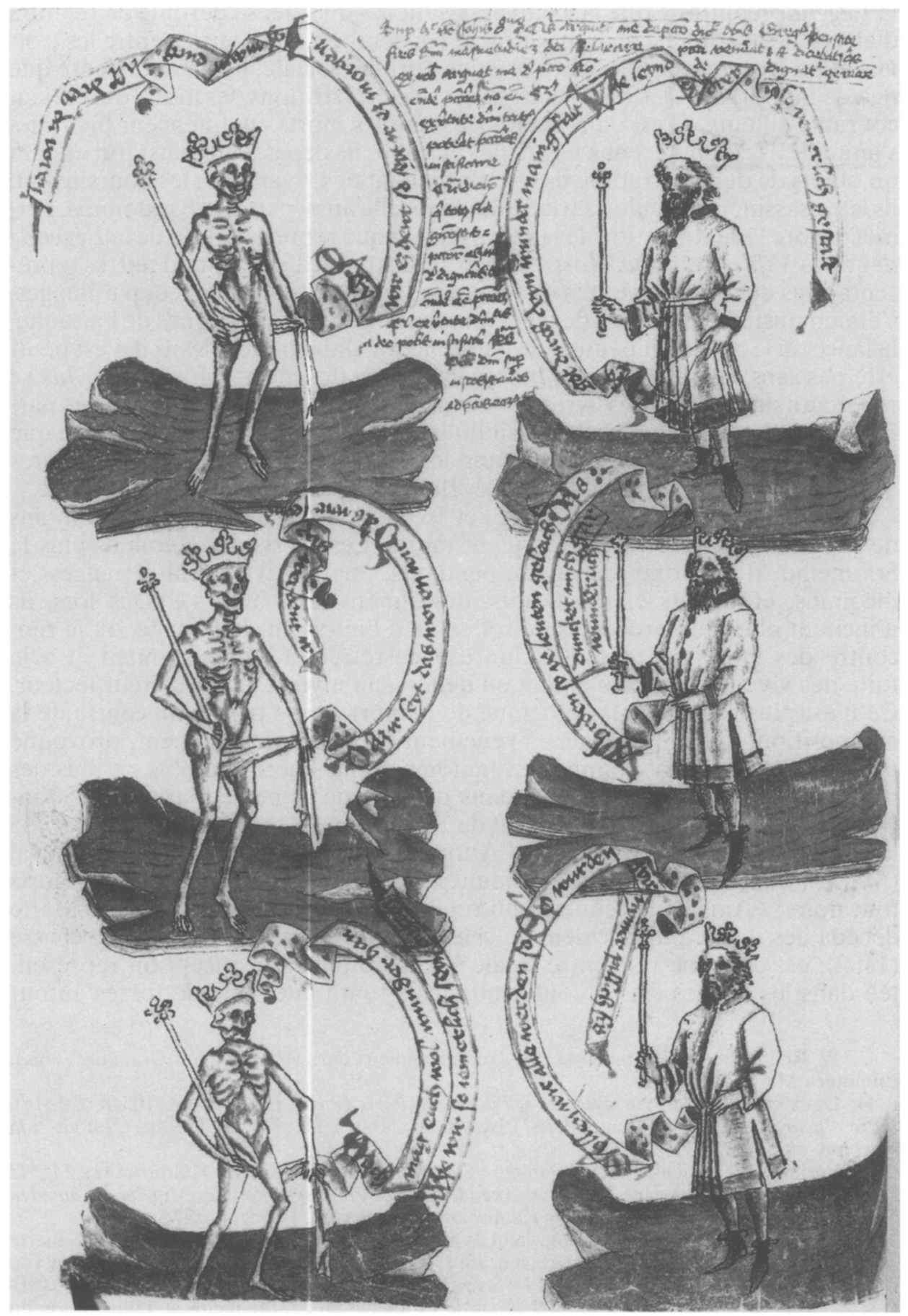




\section{IMAGES DE LA MORT, DESTRUCTION DES IMAGES AU MOYEN AGE}

la Légende manifeste que la « christianisation » du sujet se déroule en relation dialectique avec une « anarchisation ». Au début, les rencontres entre les trois vifs et les trois morts, liées parfois à une critique sociale, n'avaient encore que peu de rapport avec les récits fantastiques des apparitions des morts qui étaient courants ailleurs. C'est dans les images que les morts commencent bientôt à s'animer. Ils commencent à gesticuler et à agir, ils dépassent leur statut en tant qu'objets de démonstration, ils se rapprochent des vivants, ils les poursuivent, ils les assassinent ${ }^{43}$. Seule, la triade doublée, elle aussi parfois abandonnée, permet encore l'identification de la scène en tant que représentation de la Légende des Trois Vifs et des Trois Morts. On y voit sans doute l'influence d'autres représentations de morts agissants et agressifs. Ceux qui, dans beaucoup d'images, s'étaient justement relevés de leurs tombes portent déjà les signes de l'attaque, la lance ou le dard, signes aussi de leur fonction didactique ${ }^{44}$. Mais il n'est peutêtre pas sans signification que la transmutation des morts dans la Légende se répercute surtout dans les livres d'heures qui donnaient, malgré leur forme parfaitement chrétienne, plus de possibilités et plus de libertés à l'imaginaire que les peintures murales. C'est là, dans les marges et les bordures des livres d'heures, que la Légende garde et développe sa propre capacité subversive.

Dans les représentations des $15^{\mathrm{e}}$ et $16^{\mathrm{e}}$ siècles, il s'agit de moins en moins de rapports de symétrie entre vifs et morts. Les morts ne dévoilent plus le Soi-même transformé de leurs pendants, mais deviennent étranges et inconnus, effrayants et menaçants, deviennent les «autres ». Dès lors, ils n'incitent plus à un processus de réflexion à l'intérieur de l'image, où la rencontre des morts ne permet qu'un espace rétréci à l'ébahissement et à la fuite des vivants, mais seulement en dehors, au niveau du spectateur/lecteur. Ce n'est plus l'aspect sotériologique de la mort qui se trouve au centre de la composition, mais le caractère événementiel du dernier moment, provoqué par ceux qui, agissant comme des fantômes, sont libérés de plus en plus des traces sépulcrales. Par exemple, dans un livre de prière en latin et en français, probablement destiné à Marie de Bourgogne et terminé pour le mari de la jeune défunte, Maximilien d'Autriche, on voit une femme à cheval (Marie), accompagnée de deux jeunes nobles et attaquée par trois morts tout noirs ${ }^{45}$. Ainsi la rencontre, qui renvoie probablement au fait que Marie décéda des suites d'un accident de cheval survenu lors d'une partie de chasse (1482), est devenue rencontre finale ${ }^{46}$. Cette nouvelle conception représentée dans les images est en conformité avec toute une série de textes autour

43. W. Rotzler, op. cit., p. 218 ss. Un exemple fameux dans le Breviarium Grimani, Venise, Biblioteca Marciana, f. 449v.

44. Deux exemples: Livre d'heures à l'usage de Paris (Paris, BN, Ms. lat. 10548, f. 131v), Livre d'heures à l'usage de Rome, Paris, BN, Ms. lat. 1160, f. 151r ; cf. A. Tenenti, La vie et la mort, op. cit., ill. 8 et 9.

45. Berlin, Kupferstichkabinett (Dahlem ; Staatl. Museen Preußischer Kulturbesitz), Ms. 78 B 12, f. 220v; cf. le catalogue Zimelien. Abendländische Handschriften des Mittelalters aus den Sammlungen der Stiftung Preußischer Kulturbesitz, Wiesbaden, [1975], p. 227 f., ill.

46. Sur les imaginations qui entouraient la mort de Marie voir mon article : "Inszenierte Tode, ritualisierte Texte. Die Totenklagen um Isabella von Bourbon $(+1465)$ und Maria von Burgund (+1482) ", "Aufführung » und "Schrift » in Mittelalter und früher Neuzeit, J.-D. MüLLER éd., Stuttgart, 1996 (ibid., notes 69-71 sur le problème de l'authenticité de l'illustration du livre de prière). 
du thème de l'" everyman » qui rétrécissent plus ou moins l'espace des possibilités de l'homme quand il rencontre la Mort ${ }^{47}$. Mais, les efforts cléricaux pour maintenir le contrôle sur la mort utilisent des thèmes qui avaient déjà développé une dynamique difficile à contrôler ${ }^{48}$. La peur de la mort prématurée se joint dans un rapport complexe aux ambivalences face aux morts. Ceux-ci, mis au service du clergé, se rapprochent de l'aspect de la Mort personnifiée, mais ils gardent le caractère concret des « autres ». Devenus squelettes, désindividualisés encore plus fortement qu'avant, ils acquièrent une vitalité qui n'articule pas seulement une angoisse obscure, mais aussi un espoir curieux - celui d'une vie au-delà de la mort, d'une survivance pervertie qui se nourrit de l'agression perpétuée contre les autres, c'est-à-dire contre ceux qui sont encore à naître et à vivre.

\section{Les danses macabres}

On rencontre le même phénomène et en partie les mêmes évolutions dans les représentations des danses macabres où, sous l'influence probablement des dominicains ou des franciscains, le motif des morts dansants - appartenant certainement plus à la culture populaire qu'à celle de l'élite - est inséré dans le cadre didactique des représentations des états sociaux ${ }^{49}$. Là aussi les morts, qui entraînent des vivants ou des mourants dans leur ronde, se trouvent initialement encore au stade de décomposition $^{50}$; là aussi, le caractère d'avertissement des textes et des images est évident ; là aussi le thème garde, malgré l'enrichissement en éléments chrétiens, comme la scène prédicatoire du début, un aspect subversif ; et là aussi les morts s'animent plutôt dans les images que dans les textes ${ }^{51}$. Par exemple,

47. Encore important : K. Goedeke, Everyman, Homulus und Hekastus, Hanovre, 1865 ; en outre Th. SPENCER, Death and Elizabethan Tragedy. A Study of Convention and Opinion in Elizabethan Drama, Cambridge, 1936; Ph. S. SpINRAD, The Summons of Death on the Medieval and Renaissance English Stage, Columbus, Ohio, 1987 ; cf. aussi St. CoSACCHI, op. cit., passim; M. TITZMANN, op. cit.

48. Voir B. Roy, «Amour, Fortune et Mort : La danse de trois aveugles », Le sentiment de la mort au Moyen Age. Études présentées au Cinquième colloque de l'Institut d'études médiévales de l'Université de Montréal, C. Sutro éd., Montréal, 1979, pp. 119-137.

49. Voir A. Corvisier, «Les représentations de la société dans les danses des morts du XVe au XVIII ${ }^{\mathrm{e}}$ siècle ", Revue d'Histoire moderne et contemporaine, 16, 1969, pp. 489-538. M. LAsCAUX, La danse macabre. Images de la mort et structures mentales dans la France des XIV et $X V^{e}$ siècles, Paris, 1974 ; pour les textes allemands, le plus exhaustif est E. Koller, Totentanz. Versuch einer Textenbeschreibung, Innsbruck, "Innsbrucker Beiträge zur Kulturwissenschaft. Germanistische Reihe, 10 », 1980 ; pour les différentes traditions dans les pays européens, une bonne vue d'ensemble de manuscrits, imprimées et peintures murales demeure L. P. KuRTz, The Dance of Death and the Macabre Spirit in European Literature, New York, 1934.

50. L'idée, répandue jusqu'à nos jours, que dans les danses macabres une évolution successive aurait mené de la représentation des morts à la représentation de la Mort en tant que figure abstraite et personnifiée n'est pas convaincante : déjà dans les manuscrits français (avant Marchant), on constate l'alternance entre « la mort » et « le mort » (voir L. P. KuRTZ, op. cit., p. 27 ss). De même, affirmer que l'ambiguïté des figures est à l'origine une différenciation, parfois établie, entre «danse macabre » («Todestanz») et «danse des morts » ( Totentanz») ne paraît pas utile (St. CosACCHI, op. cit., pour exprimer cet ambiguïté, parle de «Todtentanz»).

51. Voir C. KIENING, «Totentänze - Ambivalenzen des Typus », Jahrbuch für Internationale Germanistik 27, 1995, pp. 38-56. 


\section{IMAGES DE LA MORT, DESTRUCTION DES IMAGES AU MOYEN AGE}

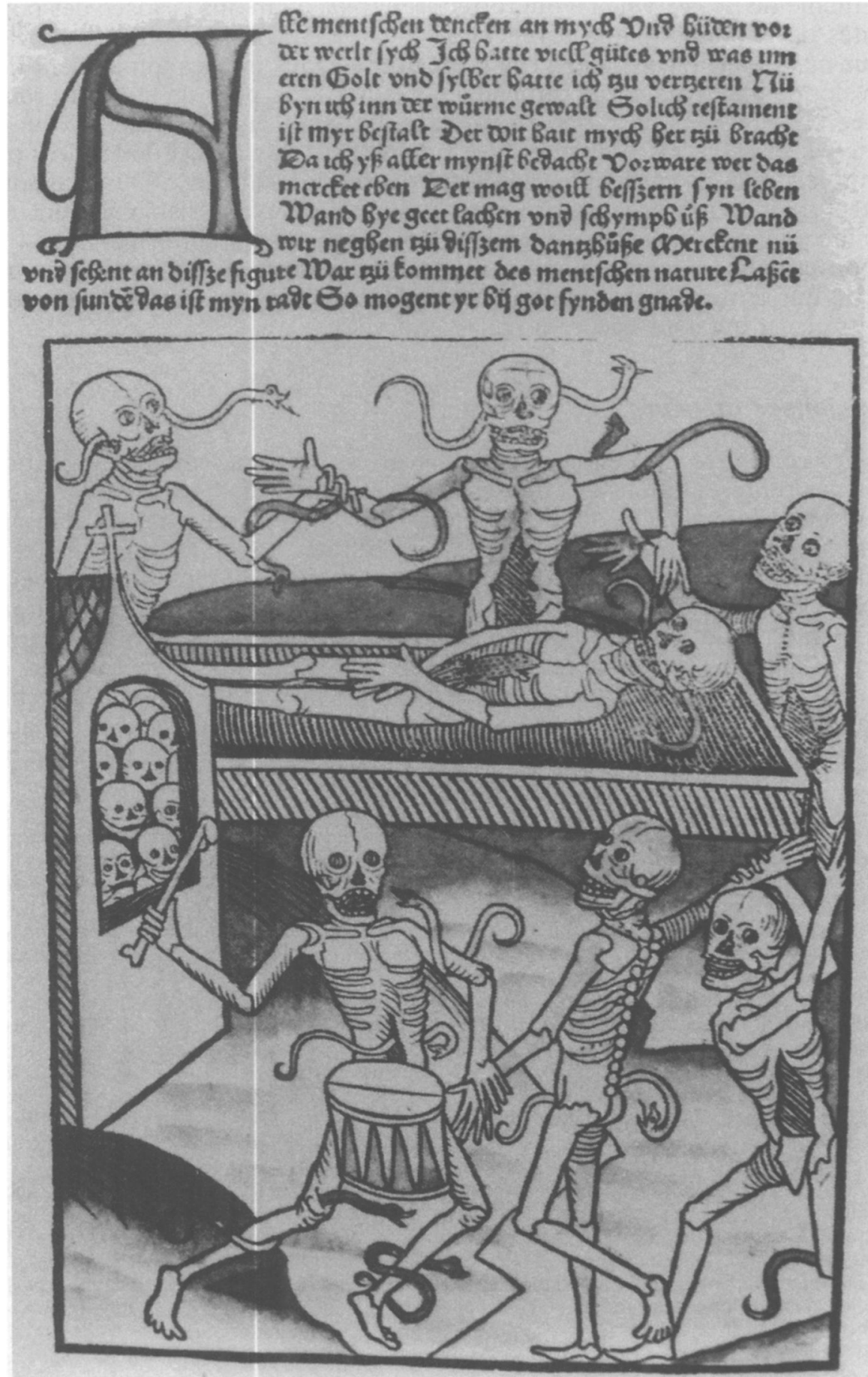




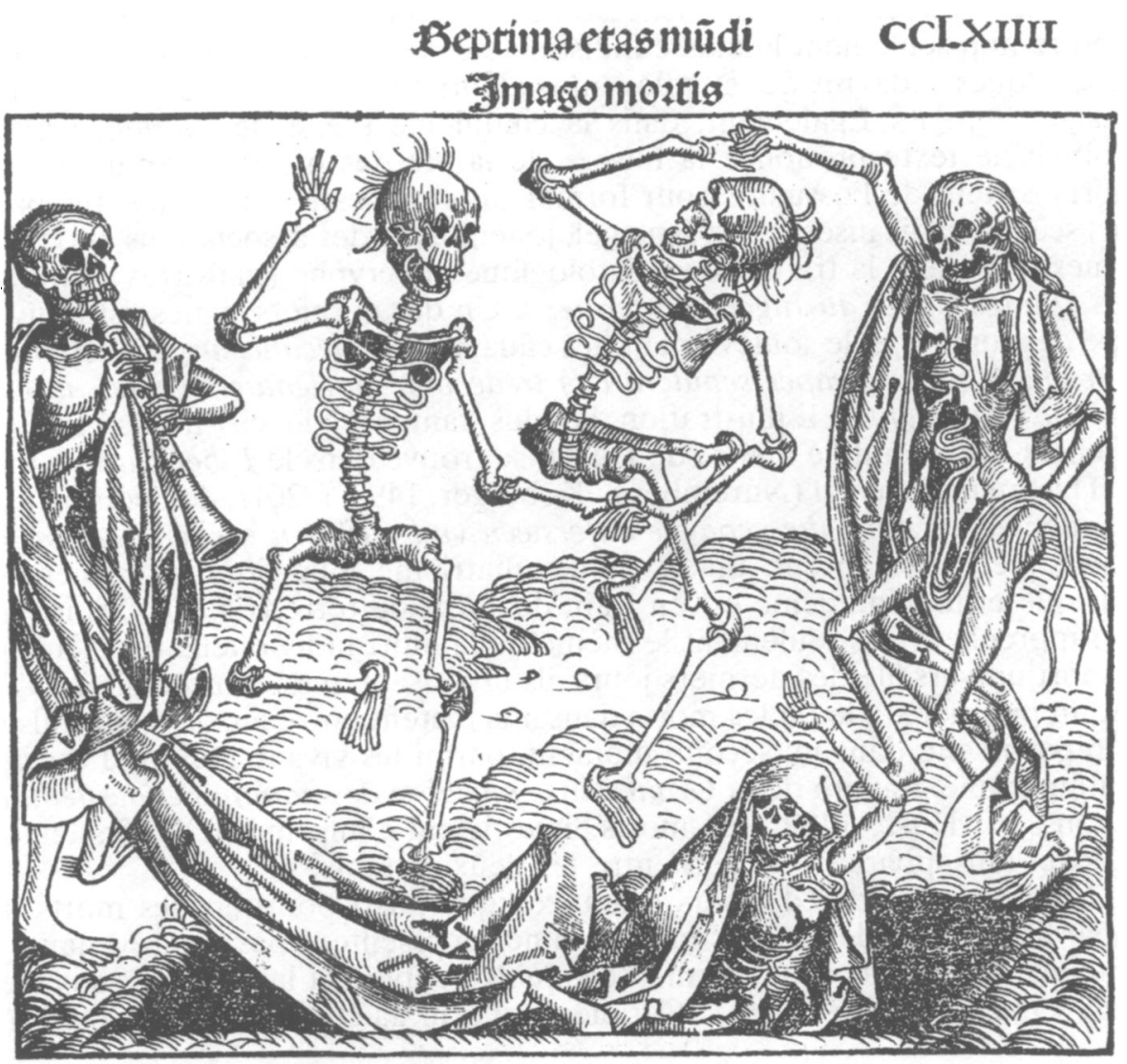

(D) 3 te mbil melius. vita int peins iniqua

Dis p̃ma mozs boim.reqes etcrna labozá

Iu fenile iugum oomino volente relaras

Elinctozúc Granes abumis ceruice catbenas

Exilinmog leuas. 2 carceris boftia frangis

Eripis indignis.iufti bona pribus equans

Etco immota manes. nulla erozabitis arte

A primo prefira bie.tu cuncta quieto

ferre iubes animo.pzomifío fine laborum

Zefine fuppliciun. $D: t \lambda$ eft $\mathrm{curcer}$ percmis

Fig. 3 ci-contre. - La danse des morts au cimetière, gravure sur bois; Der doten Danz mit figuren [Heidelberg: H. Knoblochtzer, vers 1488/89], fol. $2^{\mathrm{r}}$ (Photographie de la bibliothèque).

FIG. 4 ci-dessus. - Michael Wolgemut, La danse des morts, gravure sur bois ; Schedel, Liber chronicarum, Nürnberg : Koberger, 1493, fol. 264 (d'après Das Bild vom Tod, éd. E. SCHUSTER, Recklinghausen, 1992, p. 78). 


\section{IMAGES DE LA MORT, DESTRUCTION DES IMAGES AU MOYEN AGE}

dans la version imprimée du Mittelrheinischer Totentanz allemand (Doten Dantz mit figuren, Heidelberg: Knoblochtzer, ca. 1488-1889), le premier texte parle de l'égalité des humains qui tous vont se retrouver à l'ossuaire, lieu sans différences ni de richesse ni de beauté, tandis que l'illustration montre non seulement la masse indistincte des os, mais l'agissement bien vif de quelques cadavres qui se relèvent de leurs tombes (voir aussi la seconde image; fig. 3) ${ }^{52}$. Également, dans la cinquième scène des Simulachres de Holbein le texte prononce la misère de la vie des mortels, tandis que les morts sortent de l'ossuaire pour former un « orchestre » infernal (fig. 6a) ${ }^{53}$. Les scènes, radicalisées chez Bruegel, jouent avec des associations apocalyptiques, évoquent la tradition eschatologique apocryphe (mais très courante) des Quinze signes du Jugement dernier ${ }^{54}$. Un des derniers signes est l'ouverture des tombes et le soulèvement des cadavres : undecima surgent ossa mortuorum, et stabunt super sepulcra (...) tredecima morientur viventes, ut cum mortuis resurgant ${ }^{55}$. L'illustration la plus fameuse de ce motif, créé par Michael Wolgemut, le maître de Dürer, se trouve dans le Liber chronicarum de Hartmann Schedel (Nuremberg : Koberger, 1493, f. 261r. de l'édition allemande : Am aylften die gepayne aufersteen vnd auff den grebern stehen) où trois squelettes dansent, tandis qu'un quatrième joue d'un instrument de musique et un cinquième est en train de sortir de terre (fig. 4). Ces images ne rapprochent pas seulement le thème de la mort individuelle du thème de la mort universelle des derniers jours, ils favorisent aussi l'impression qu'il y eut un premier stade où les morts dansants restent entre eux, avant qu'ils ne se répartissent, dans un second moment, parmi les vivants, tel qu'il est présenté par une gravure de la Grant danse macabre des hommes et des femmes (Lyon: M. Husz, 1499; les morts investissent l'imprimerie) ${ }^{56}$. Seulement Bruegel fait apparaître le lien entre les deux instants.

L'arrière-plan des derniers jours explique aussi pourquoi les morts qui apparaissent dans les danses macabres ne représentent pas des revenants et ceux qui sont morts avant terme, tels qu'ils peuplaient la " chasse sauvage " ou la «messenie Herlekin ${ }^{57}$. Car même si ceux-là étaient parfois figurés (et

52. Fac-similé par A. Schramm, Leipzig, 1922 ; cf. aussi Der tanzende Tod. Mittelalterliche Totentänze, G. KAISER éd., Francfort, 1983 (insel taschenbuch, 647), p. 192 ss.

53. Dans les épreuves des gravures, garnies avec des titres allemands, l'image des morts (titre : «Gebeyn aller menschen ») se trouvait, semble-t-il, à la fin, directement avant le jugement dernier; voir H. Massmann, op. cit., p. 7, et l'édition des épreuves : Die Todesbilder und das Todesalphabet von Hans Holbein, Berlin, 1922. Sur le caractère ambigu de l' orchestre " des morts R. Hammerstein, Tanz und Musik des Todes. Die mittelalterlichen Totentänze und ihr Nachleben, Berne-Munich, 1980, pp. 93-95.

54. Sur les textes français cf. Dictionnaire des lettres françaises. Le Moyen Age, G. HASENOHR et M. ZINK éds, Paris, 1992, p. 1217 ss.

55. Pierre le Mangeur, Historia scholastica, éd. Migne, Patrologia latina, t. 198, Paris, 1855, p. 1611 ; d'autres passages dans E. KOlleR, op. cit., p. 403 ss.

56. L. Febvre et H.-J. Martin, L'apparition du livre, $2^{\mathrm{e}}$ éd., Paris, 1971, p. 89 (ill.).

57. Voir O. HöFLER, Kultische Geheimbünde der Germanen, Francfort, 1934 ; J.-C. SCHMITT, "Les "superstitions" », Histoire de la France religieuse, J. LE GoFf et R. RÉmond éds, Paris, 1988 , p. 524 ss. Dans une seule représentation de la Légende, créée probablement dans l'atelier de Dürer, les trois morts, volants, se rapprochent de la chasse sauvage (Vienne, Albertina; J. WIRTH, op. cit., fig. 21). De même peut-on penser au type italien de la furie de la Mort. 
assez tardivement) sous l'aspect de cadavres ou de squelettes ${ }^{58}$, la relation n'est pas réciproque. De même, ni dans la Légende, où les morts parlent quelquefois de leur vie passée, ni dans la danse macabre, où les morts se réfèrent le plus souvent à la vie passée de leurs interlocuteurs, ne se présentent de pauvres âmes souffrant les peines du purgatoire. Ceux qui se relèvent ici de leurs sépultures sont tous ceux qui ont déjà parcouru le cheminement de la vie terrestre, les «autres» par excellence. Ils se mêlent pourtant avec d'autres apparitions des morts sous la forme de cadavres, par exemple avec les ancêtres qui occupent le pouvoir par leur simple présence (tels que Dürer les représentait dans une illustration du Ritter vom Turn) ${ }^{59}$; ils peuvent aider les vivants mais ils possèdent de plus en plus des traits persifleurs, sinon violents.

Dans les danses macabres avant Holbein les morts ne sont pas munis d'armes, seulement (pas toujours) d'instruments de musique ${ }^{60}$. Leur pouvoir se définit à travers l'irrésistibilité. Il consiste en la simple conscience de l'avenir des vivants, une conscience qui se fonde sur le fait paradoxal qu'ils représentent eux-mêmes l'avenir qu'ils annoncent. Il consiste aussi en une certaine "autonomie de l'anonymat », au-delà de l'identité. Les morts forment une communauté entre eux, une communauté toujours grandissante. Ils ne s'y présentent pas comme intermédiaires de Dieu; ils apparaissent, jouent des instruments de musique (comme dans une gravure ajoutée dans la seconde édition de la Danse macabre de Guyot Marchant, Paris, 1486) ${ }^{61}$ et dansent - sans être appelés, sans avoir un passé ou un avenir. Ils inquiètent justement par une vitalité à la fois humaine et inhumaine. Déjà le manuscrit de Kassel (Badische Landesbibliothek, $4^{\circ}$ Ms. poet. et roman. 5 ; vers 14601470) du Mittelrheinischer Totentanz offre des illustrations très vivantes, influencées par l'enluminure dite «bourguignonne », où les morts ont pris les attributs de leurs «partenaires », où ils sautillent et font des acrobaties, où ils prétendent jouer de la musique avec quelques os et témoignent ainsi de l'augmentation du pouvoir anarchique de la majorité écrasante des morts qui est illustré dans l'incunable (Doten Dantz mit figuren) un peu plus tard.

Ici comme dans toutes les danses macabres avant Holbein, la plupart des illustrations, à l'exception de celles du début et de la fin, se concentrent sur des couples de figures et sur des rapports de symétrie entre les morts et ceux qui doivent mourir. Les derniers, à qui n'est accordé aucun répit pour changer leur mode de vie, doivent s'insérer à la masse amorphe des morts, se ranger parmi les « hommes noirs », les « compagnons » ou les « apparitions » de la Mort ${ }^{62}$. Chaque homme rencontre son propre «partenaire de danse » qui, par ses attributs, se présente en même temps comme son propre reflet dis-

58. Voir J. Wirth, op. cit., p. 109 ss à propos du Retable des Pauvres Ames (1505; Berne, Musée d'Art), fig. 88/89; Himmel, Hölle, Fegefeuer, op. cit., pp. 21, 131; autres exemples chez J.-C. SCHMITT, op. cit., p. 244 ss.

59. J. WirTh, op. cit., fig. 15 ; un autre exemple chez P. M. HALM, «Altbayerische Totendarstellungen », Münchner Jahrbuch für bildende Kunst, 4, 1909, pp. 143-159.

60. L'étude fondamentale de cet aspect est celle de R. HAMMERSTEIN, op. cit.

61. Ibid., pp. 72-74.

62. Oberdeutscher vierzeiliger Totentanz (d'après le livre xylographique ; Heidelberg, Bibl. univ., Cpg 438, vers 1465) ; le patriarche ( $\left.n^{\circ} 5\right)$ : « Nw wil mich der tod twingen / Mit seynen geseln zu springen »; l'évêque : « Nw czyen mich dy vngeschaffen / Czu dem tode als eynen 


\section{IMAGES DE LA MORT, DESTRUCTION DES IMAGES AU MOYEN AGE}

tordu ${ }^{63}$ et qui peut justement par cette rencontre individuelle s'approcher de l'image personnifiée de la Mort. La transition entre les morts et la Mort personnifiée y adopte des formes multiples. Pour l'enfant qui se plaint dans le Oberdeutscher vierzeiliger Totentanz qu'un homme noir l'enlève ("Eyn swarzer man zeut mich do hyn ") ${ }^{64}$, la Mort n'est pas le Soi-même transmuté par le temps, mais l'autre, l'étranger. Cependant, cette Mort, masculine en allemand ${ }^{65}$, reste d'une manière particulière aussi sa propre mort, c'est-àdire l'homme noir des contes de nourrices et des fantasmes enfantins ${ }^{66}$.

L'opposition de la Mort à l'enfant indique une dissociation croissante entre les morts et les vivants. Elle se manifeste clairement dans la danse macabre bernoise de Niklaus Manuel du début du $16^{\mathrm{e}}$ siècle ${ }^{67}$. Les attributs reliant la Mort à son interlocuteur n'y sont plus l'expression d'une identité cachée entre morts et vivants, mais plutôt celle d'une activité de la Mort, de sa capacité à se rapprocher de l'homme dans une ressemblance persiflante. Le pouvoir, qui s'y dévoile, ne repose plus sur les sautillements moqueurs des morts mais sur une dérision profonde qui attaque les hommes intimement. Les deux scènes qui démontrent cela de la façon la plus impressionnante, sont celle où la mort (masculine) touche lubriquement les seins de la jeune fille (fig. 5a) et celle (la scène finale) où la mort se glisse près du peintre lui-même pour lui enlever le pinceau de la main (fig. 5b) - un motif que Vauzelles, dans la préface des Simulachres, reprendra au regard de la fin de Lützelburger. Chez Manuel, les vers joints à la dernière scène se tournent d'une façon traditionnelle vers le Christ, mais l'impression persiste que le jeu avec la vivacité croissante des morts / de la Mort puisse finalement aussi bien amener à la remise en question de la conception chrétienne de la mort comme transitus ${ }^{68}$. Car si l'instant du décès, suivi du jugement particulier (institutionnalisé par la bulle de Benoît XII du 29. 1. 1336), est le plus souvent compris comme moment décisif pour le salut ou la damnation, la Mort, acteur de la dernière heure, acquiert un pouvoir qui ressemble à celui du diable ${ }^{69}$. Déjà dans la troisième image de Manuel, où la Mort, redressée

affen ». La Mort elle-même désigne les squelettes comme «frères noirs » (en parlant au roi), « singes » (à l'archevêque) ou « loups sauvages » (au comte).

63. Le duc dans la Danse macabre de Bâle (d'après les estampes de Merian, Francfort, 1649, p. 61) regrette : « Jetzund werd ich meim Täntzer gleich »; de même l'abbé (p. 67) : «Dennoch bin ich dem Todt gleich ».

64. Ibid., $\mathrm{n}^{\circ} 23$ (numérotation de l'original) ; cf. Der tanzende Tod, op. cit., p. 382.

65. Voir J. BialostocKI, «Das Geschlecht des Todes. Symbolbildung und Sprache », Mnemosyne. Festschrift für Manfred Lurker zum 60. Geburtstag, W. BIEs et H. JuNG éds, BadenBaden, «Bibliographie zur Symbolik, Ikonographie und Mythologie. Ergänzungsband, 2 », 1988, pp. 109-132.

66. Sur cette imagination cf. déjà K. J. SCHRÖER, « Todtentanzsprüche », Germania, 12, 1867, pp. 286-288.

67. Berne, Historisches Museum ; Reproduction des images (conservées seulement dans les copies de Albrecht Kauw de 1649) avec explication par P. ZinsLi, 2e éd., Berne, "Berner Heimatbücher, 54/55 », 1979.

68. G. Martelet, Victoire sur la mort. Éléments d'anthropologie chrétienne, $2^{\mathrm{e}}$ éd., Paris 1963 ; A. M. HAAs, Todesbilder im Mittelalter. Fakten und Hinweise in der deutschen Literatur, Darmstadt, 1989, part. pp. 26-49.

69. Pour le jugement particulier voir l'article de P. ADNÈs, Dictionnaire de spiritualité, 8, 1974, col. 1581-1585; et le catalogue de l'exposition Himmel, Hölle, Fegefeuer. Das Jenseits im Mittelalter [catalogue : Peter JEZLER], Zurich, 1994, p. 18. 
fièrement, désigne le Christ à la croix avec son doigt tendu, l'impression reste ambiguë : l'indication de la victoire sur la mort s'exprime par un geste de pouvoir de la Mort. De telles ambivalences favorisaient sans doute l'usage de la figure squelettique pour des intentions critiques face à l'Église et aux pouvoirs politiques ${ }^{70}$.

\section{Le double, l'autre et le Soi-même}

A l'époque de Manuel, les expérimentations avec le nouveau pouvoir de la Mort sont déjà arrivées à un stade avancé comme expérimentations avec le double, auquel on suppose une appropriation élargie du monde humain. Ce qu'on rencontre dès lors plus souvent comme motif, c'est la capacité de la Mort / des morts de se dissimuler et de se travestir, de se masquer et d'effrayer les vivants en soulevant les déguisements ${ }^{71}$. Ces jeux de dissimulation et de travestissement ne se subordonnent que superficiellement à l'angoisse d'une mort prématurée et inattendue, survenant au plein cœur de la vie quotidienne. Ils expriment plutôt une violence archaïque, le pouvoir des identités fluctuantes des morts, face aux identités inamovibles des vivants.

Peu importe s'il s'agit des figures de la Mort ou des morts, les doubles qui apparaissent dès la fin du $15^{\mathrm{e}}$ siècle représentent une nouvelle réalité et une nouvelle présence. Ils sont les absolument «différents » qui n'apparaissent plus seulement sur des lieux particuliers, mais qui se mêlent aux vivants qu'ils copient. Même dans les manuels d'anatomie, qui accentuent l'authenticité des représentations des squelettes, les figures démonstratives, normalement privées de vie, sont équipées de traits vivants : ainsi André Vésale montre un squelette, semblable à celui de Bruegel un peu plus tard, dans une pose pensive - absorbé par la contemplation d'un crâne ${ }^{72}$. Les squelettes deviennent de plus en plus des imitations distordues qui commencent à réfléchir (comme chez Bruegel) et à désirer. Un point extrême est atteint dans les tableaux et esquisses qui sont le plus souvent regroupés sous le titre 4 la jeune fille et la mort ${ }^{73}$. La mort y est devenue le contraire d'un reflet de miroir, l' «autre » par excellence, une figure masculine qui exprime des fantasmes masculins. Au-delà d'une simple morbidité perverse, cette Mort masculine, sujet et parfois aussi objet du désir ${ }^{74}$, est le signe de l'inscription de la «contre-force » à l'intérieur de la vie elle-même. Justement la forme la plus puissante, la présence la plus intime, la tactilité la plus forte de la Mort la démontre absorbée par le Soi-même, par l'entre-

70. C.-A. BEerli, Le peintre poète Nicolaus Manuel et l'évolution sociale de son temps, Genève, Université de Genève, Faculté des Sciences économiques et sociales, 1953, thèse $\mathrm{n}^{\circ} 139$.

71. Pour la littérature on trouve des aspects intéressants dans les actes du colloque Masques et déguisements dans la littérature médiévale, M. L. Ollier éd., Montréal-Paris, 1988.

72. André VÉsale, De humani corporis fabrica, Bâle : Oporin, 1543, p. 204 ; cf. P. AriÈs, Images de l'homme devant la mort, Paris, 1983, fig. 264.

73. J. WirTH, op. cit., pp. 80-93.

74. Dans un clair-obscur de Manuel (1517; Bâle, Musée d'Art) au revers d'une représentation de David et Bethsabée; cf. J. WirTh, op. cit., fig. 69 ; P. ARIĖs, op. cit., fig. 258. 


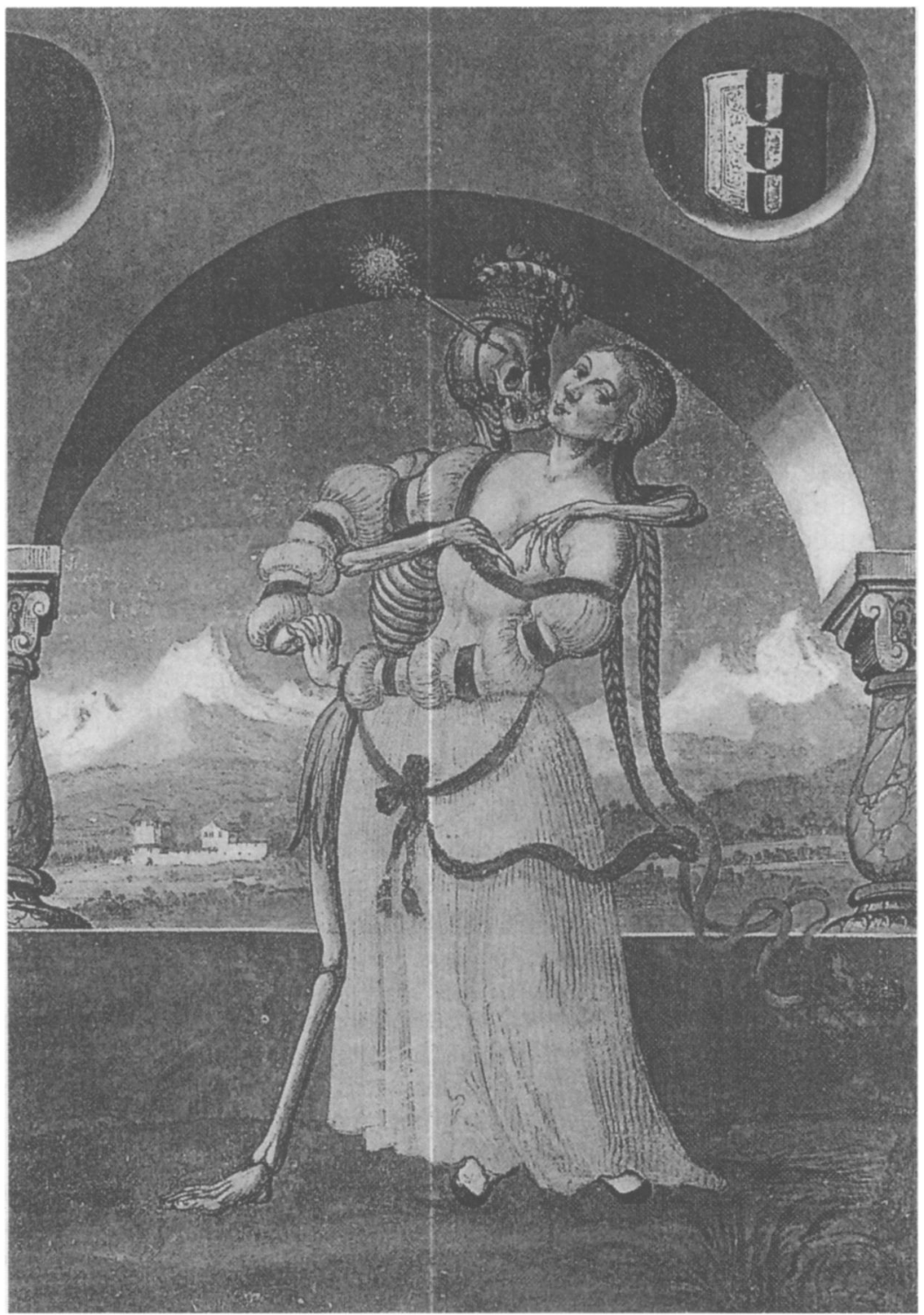

Fig. 5. - Niklaus Manuel' (dit Deutsch), La Danse Macabre de Berne (1516-1519, copie en aquarelle de Albrecht Kauw de 1649 ; d'après Das Bild vom Tod). a. La mort et la jeune fille. 


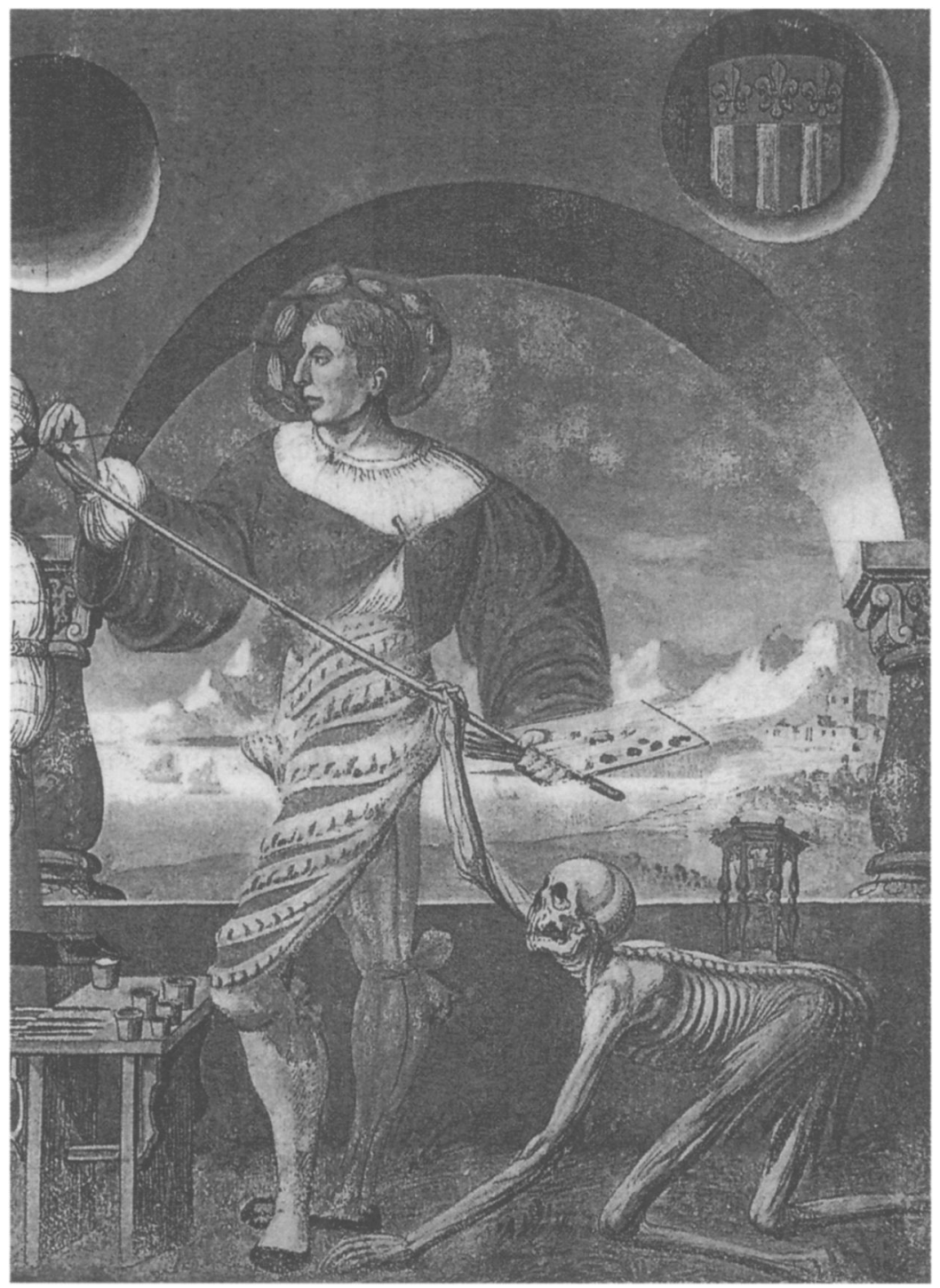

Fig. 5b. - La mort et la peintre ; ibid. (p. 83). 


\section{IMAGES DE LA MORT, DESTRUCTION DES IMAGES AU MOYEN AGE}

mêlement d'angoisse et de désir. Ainsi le moment de la prise de conscience, illustré par exemple à travers un regard dans le miroir qui dévoile une figure tordue, devient central ${ }^{75}$. Dans une eau-forte de Daniel Hopfer, la dame, comme envoûtée, fixe le miroir, tandis que la servante ne peut pas croire ce qu'elle y voit : un crâne, tenu par la Mort, le compagnon du diable ${ }^{76}$. Pareillement, la dame noble de la Danse macabre de Bâle est fascinée par son alter ego qui se reflète fugitivement dans le miroir ${ }^{77}$. Dans chacun de ces cas, la Mort représente un Soi-même déplacé dans le temps et dans l'espace, aperçu momentanément comme un fantôme. Ce n'est qu'au niveau d'un spectateur entraîné à la dialectique de la présence et de l'absence face à la mort que les différents personnages et différents regards se rejoignent. C'est là où le déplacement entre l'homme et le cadavre, la fluctuation de la figure squelettique entre tout et rien, réalité et fantôme, présence subjective et présence objective peuvent être aperçus.

Ainsi on découvre deux mouvements complémentaires et dialectiques dans l'histoire des rencontres entre l'homme et le cadavre ou le squelette : d'une part, le processus par lequel les morts, représentant de moins en moins des reflets simplement déplacés dans le temps, deviennent de plus en plus les " autres », les ennemis ; d'autre part, le processus qui infuse la mort dans l'existence elle-même, qui l'inscrit dans la conscience. A la «conceptualisation » et à l'«extériorisation » de la mort sur le plan de l'image correspond son «intériorisation»sur le plan psychologique ${ }^{78}$.

Les deux mouvements se laissent poursuivre clairement dans les gravures de Holbein qui marquent mieux que bien d'autres l'évolution qui mènera jusqu'au Triomphe de la Mort de Bruegel. Holbein, qui a traité le sujet à peu près en même temps dans l'Alphabet de la Mort, utilisé dans des imprimés bâlois dès 1524, et dans les Simulachres (tous les deux gravés par Hans Lützelburger), n'a pas créé une autre danse macabre, mais une série de scènes de la vie quotidienne où la Mort / les morts interviennent toujours de nouveau $^{79}$. Cette série est constituée à la base d'un ordre sotériologique qui commence avec la création (dans l'Alphabet : l'orchestre des morts ; fig. 6a) et se termine avec le jugement dernier. Les figures squelettiques cependant, qui apparaissent à partir de l'expulsion du paradis, ne fonctionnent pas vraiment en tant qu'intermédiaires de Dieu. Ils agissent plutôt d'une façon autonome, et le pouvoir de la Mort se prolonge même au-dessus du jugement : dans la dernière gravure ( $\mathrm{n}^{\circ} 41$ de l'édition de 1538 ; fig. $6 \mathrm{f}$ ), la Mort est réduite au signe de la mortalité, représentée par une tête décharnée au milieu d'un blason, mais elle lève ses deux bras squelettiques au-dessus du

75. Voir F. Bächtiger, op. cit., p. 59 ss, et G. F. Hartlaub, Zauber des Spiegels, Munich, 1951, pp. $149-158$; cf. aussi n. 5.

76. J. WIRTH, op. cit., fig. 75.

77. Der tanzende Tod, op. cit., p. 232 ss (d'après l'édition de Merian, 1649, p. 81).

78. Voir M. Foucault, Folie et déraison. Histoire de la folie à l'âge classique, Paris, 1961 , p. 19: «L'effroi devant cette limite absolue de la mort s'intériorise dans une ironie continue ».

79. K. HoffmANN, « Holbeins “Todesbilder". Laienfrömmigkeit im Todesbild der Vor- und Frühreformation ", Laienfrömmigkeit im späten Mittelalter. Formen, Funktionen, politischsoziale Zusammenhänge, K. SCHREINER éd., Munich, « Schriften des Historischen Kollegs. Kolloquien, 20 ", 1992, pp. 263-282, constate une " "Implosion" des Todes in die Lebenswirklichkeit »(p. 267). 
blason et tient une pierre, expression de son caractère « fondamental », dans ses mains ${ }^{80}$. Le couple patricien, qui se présente avec son blason, renvoie au péché originel, qui a donné naissance à la mort, et constitue un lien entre les images des squelettes agissants et la réflexion personnelle du spectateur ${ }^{81}$.

Le caractère dynamique et transgressif de la figure de la Mort se révèle chez Holbein particulièrement dans le motif central de la folie et du déguisement ${ }^{82}$. Les morts dévoilent d'abord, comme on a vu, une nouvelle capacité d'assimilation. Ils donnent à chacun sa propre mort : l'astronome («Sternensecher »), par exemple, est confronté avec un crâne, c'est-à-dire avec le signe de la mortalité avec lequel il est familier (fig. 6c). Ils prennent aussi des rôles de la vie quotidienne : ceux du servant, du musicien, du guerrier, du bouffon. Mais ils ne les prennent qu'à la façon d'un jeu de masques, transparent pour les spectateurs et parfois aussi pour les personnes à l'intérieur de l'image : c'est un effet de choc pour le groupe galant quand la Mort-fou se retourne et dévoile son aspect véritable ( $\mathrm{n}^{\circ} 11$; fig. $\left.6 \mathrm{~b}\right)$. Le contraste entre le comique et le dramatique est ainsi pénétré par la proximité intime entre la mort et la folie qui, sous l'influence surtout de La nef des fous de Brant/ Dürer, se manifeste dès la fin du $15^{\mathrm{e}}$ siècle. Dès lors, les figures de la Mort et du bouffon/fou se rejoignent souvent dans les images et se superposent dans les connotations de la vanité : Hans Beham, par exemple, qui a montré dans une gravure de 1540 un fou courtisant une femme, fait apparaître dans une reprise presque identique de la gravure, un an plus tard, sous le bonnet de bouffon une tête décharnée, tandis qu'une phrase ajoutée se réfère à la vétusté $^{83}$. Le fait que la différence se réduise à peu entre la Mort et le fou est dû, d'un côté, à l'intention didactique, qui stigmatise l'absence de la meditatio mortis comme folie, et, de l'autre côté, au paradoxe de la folie elle-même qui représente à la fois une aberration et la normalité : chez Brant, le monde

80. Une interprétation de l'image est donnée par F. BÄCHTIGER, op. cit., pp. 114-122, qui souligne l'aspect de la durabilité et stabilité de la pierre. Il semble cependant que là où Bächtiger voyait un «creux » ("Vertiefung ») en bas du bord de l'image, il s'agit plutôt des lacunes dans le soubassement dont la pierre (dans les mains de la « Mort ») provient. Vauzelles s'est inspiré de l'image pour développer, dans le texte commençant justement sur la page en face («Figures de la Mort »), le motif d'une pierre angulaire en tant qu'obstacle aussi bien que signe de l'égalité : «Car aux cheminans quelque foys se rencontrent les pierres, \& par l'offendicule quelles font aux piedz, souuent sont trebucher les gens. Qui nous figure la Mort, qui ainsi a l'improueu les cheminans tant plus rudement frappe, \& prosterne, d'autant qu'elle les trouue plus asseurez, \& et non aduisez. Or la pierre angulaire est faicte en sorte, que en quelque sorte qu'elle tombe, elle demeure droicte, a cause de son equalité. Aussi la Mort pareillement esgallement tombante, esgalle aussi toutes puissances, richesses, haultainetez, \& delices en vng coup les desrompant. » (Simulachres, $\mathrm{Hi}^{\mathrm{r}}$ ).

81. F. BäCHTIGER, op. cit., p. 120 ss.

82. Voir C. Blum, «La folie et la mort dans l'imaginaire collectif du Moyen Age et du début de la Renaissance (XII ${ }^{\mathrm{e}}-\mathrm{XVI} \mathrm{I}^{\mathrm{e}}$ siècles). Positions du problème », Death in the Middle Ages, H. Braet et W. Verbeke éds, Louvain, «Mediaevalia lovaniensia. Series 1, Studia 9 », 1982, pp. 258-285 ; T. Schwarz, «Der Tod im Gewande des Narren. Zur Verbindung von Narrenidee und Vanitas-Thematik in Wort- und Bildzeugnissen des 15.-17. Jahrhunderts ", Narren, Schellen und Marotten, W. MezGer éd., 2e éd., Remscheid, 1984, pp. 387-411; W. MEZGER, Narrenidee und Fastnachtsbrauch. Studien zum Fortleben des Mittelalters in der europäischen Festkultur, Constance, « Konstanzer Bibliothek, 15 », 1991, pp. 419-466.

83. Nürnberg, Germanisches Nationalmuseum, Kupferstichkabinett, STN 614 et 615 ; J. Wirth, op. cit., fig. 141-142; W. MeZger, Narrenidee, op. cit., p. 430 ss, ill. 234a/b. 
IMAGES DE LA MORT, DESTRUCTION DES IMAGES AU MOYEN AGE

Vx væ væe habitantibus in terra.

A P OCALYPSIS VIII

Cuncta in quibus fpiraculum vitæ eft, mortua funts GE NESIS VII

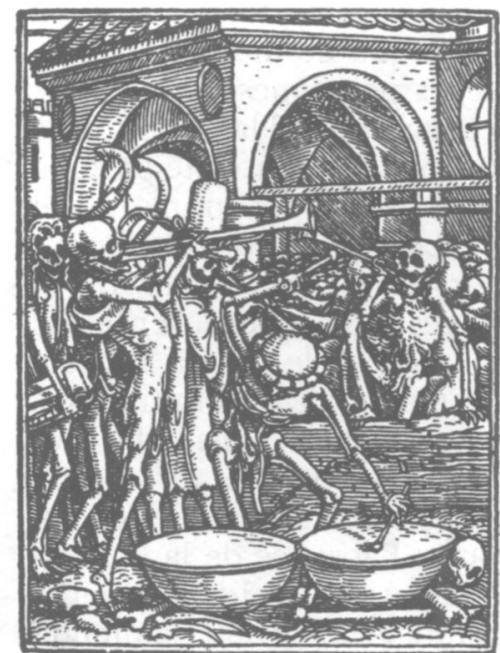

Malheureux qui uiuez au monde Toufiours remplis d'aduerfitez, Pour quelque bien qui uous abonde, Serez tous de Mort uifitez.

FIG. 6. - Holbein/Lützelburger, Images de la Mort, gravures sur bois ; Les simulachres \& historiees faces de la mort, Lyon: Trechsel, 1538 (d'après le fac-similé, New York, 1971).

a. L'« orchestre » des morts (p. $\left.20 ; n^{\circ} 5\right)$.
Mulieres opulentre furgite, \& audite vocem meam.Poft dies, $\&$ annum, $\&$ vos conture bemini.

$$
\text { IS II } X \times X I I
$$

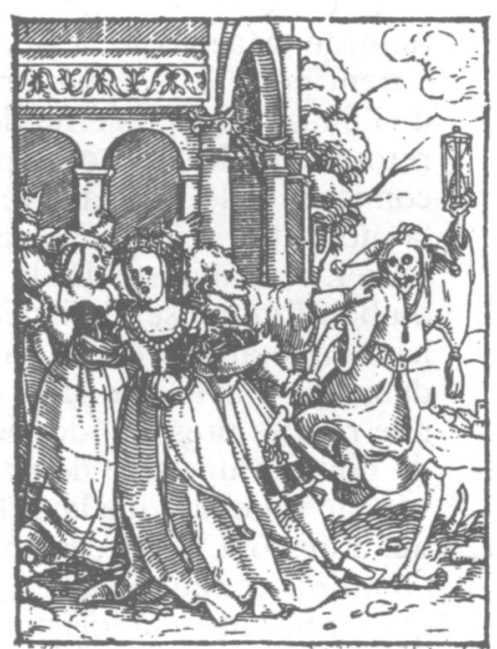

Leurz uous dames opulentes. Ouyez la ioix des trefpaffez. Apres maintz ans $\mathcal{X}$ iours paffez, Serez troublées \& doulentes.

b. La Mort comme bouffon (p. $\left.26 ; \mathrm{n}^{\circ} 11\right)$. 


\section{Indica mihi fi nofti omnia.Sciebas quodd nafciturus effes, \& numerum dierum tuorum nouerase}

$$
\text { IO B XXVIII }
$$

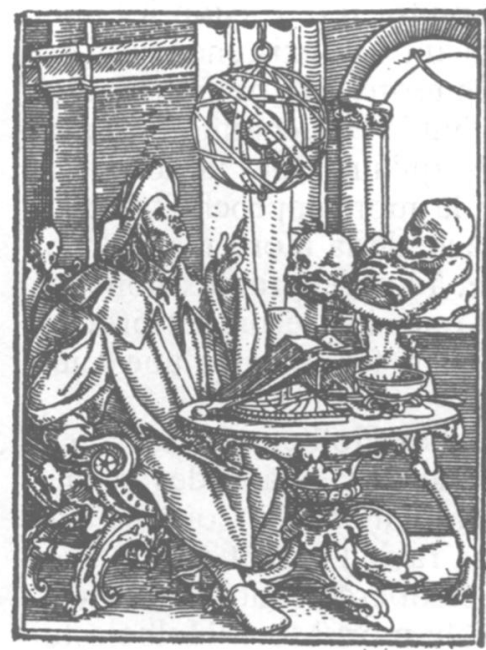

\section{Tu dis par Amphibologie}

Ce qua aux aultres doibt aduenir.

Dy moy donc par Aftrologie

\section{Quand tu deburas a moy uenir"}

\section{Qui congregat thefauros mendacï vanus $\mathcal{Q}$ excors eft, \& impingetur ad laqueos mortis.}

$$
P R O V E R \cdot \quad \times X I
$$

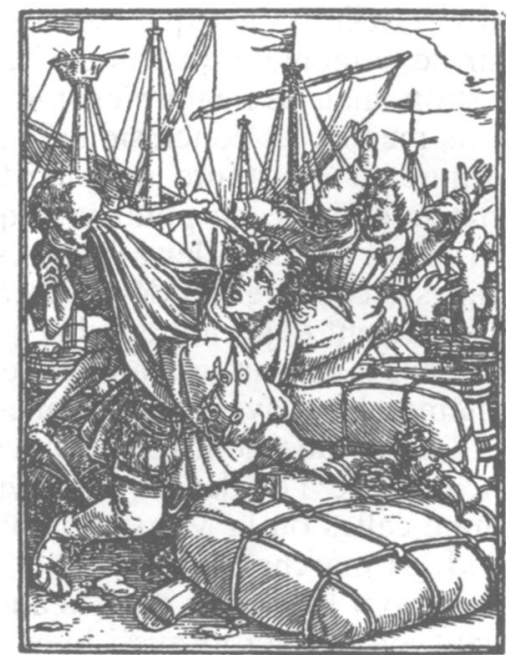

\section{Vain eft cil qui amaffera} Grand $z$ biens, $\&$ trefors pour mentir, La Mort l'en fera repentir. Car en fes lacz furpris fera.

c. La Mort et l'astronome (p. $\left.42 ; n^{\circ} 27\right)$.

d. La Mort et les marchands (p. 44 ; $n^{\circ} 29$ ).

est rempli de fous qui ne sont plus des figures de la limite, mais des miroirs distordus dans lesquels la société se reflète ${ }^{84}$.

Pourtant, la mise en scène de l'ensemble de la Mort et du fou n'est pas sans ambiguïté. Elle ne rappelle pas seulement la réciprocité de vie et de mort selon le «contrat » entre l'ici-bas et l'au-delà (une vie déraisonnable mène à une mauvaise mort et à la perte du salut), elle fait aussi penser que la mort elle-même est l'aspect de la folie. Ainsi, le fou, chez Brant/Dürer celui qui a insensément tenté de fuir la (méditation de la) mort ${ }^{85}$, est chez Bruegel

84. Sur les fonctions et ambiguités du fou, avant Brant, voir le bon livre de J.-M. FrITZ, Le discours du fou au Moyen Age. XII'-XIII siècles. Etude comparée des discours littéraire, médical, juridique et théologique de la folie, Paris, 1992.

85. Première édition allemande : Bâle, Bergmann von Olpe, 1494, $\mathrm{n}^{\circ} 85$ : « Nit fursehen den dot ». 


\section{IMAGES DE LA MORT, DESTRUCTION DES IMAGES AU MOYEN AGE}

celui qui renonce à se révolter insensément contre la masse des morts : il se cache sous la table justement en fixant l'occiput d'un squelette masqué (fig. 1 ). Son regard et son cri indiquent la prise de conscience, au moins le pressentiment, que c'est la mort qui sous les masques de la vie, l'étrange qui sous l'apparence de l'ordinaire, et la folie qui sous le manteau de la raison, se sont déjà instillés partout. Michel Foucault en a conclu à la constitution d'un nouveau discours de la folie : «La substitution du thème de la folie à celui de la mort, écrit-il, ne marque pas une rupture, mais plutôt une torsion à l'intérieur de la même inquiétude. C'est toujours du néant de l'existence qu'il est question, mais ce néant n'est plus reconnu comme terme extérieur et final, à la fois menace et conclusion; il est éprouvé de l'intérieur, comme la forme continue et constante de l'existence. Et tandis qu'autrefois la folie des hommes était de ne point voir que le terme de la mort approchait, tandis qu'il fallait les rappeler à la sagesse par le spectacle de la mort, maintenant la sagesse consistera à démontrer partout la folie, à apprendre aux hommes qu'ils ne sont déjà rien de plus que des morts, et que si le terme est proche, c'est dans la mesure où la folie devenue universelle ne fera plus qu'une seule et même chose avec la mort elle-même ${ }^{86}$.

L'inquiétude dont parle Foucault est nourrie chez Holbein par les alternances entre l'évidence et l'invisibilité des squelettes ${ }^{87}$. Dans des scènes comme celles où la Mort apparaît à quelques clercs $\left(n^{\circ} 12,14\right)$, où elle surprend les marchands ( $\mathrm{n}^{\circ} 28 / 29$; fig. $6 \mathrm{~d}$ ), où elle ravit l'enfant ( $\left.\mathrm{n}^{\circ} 39\right)$, les vivants sont horrifiés; dans beaucoup d'autres ils n'aperçoivent pas la Mort. Jouant avec le statut aussi bien réel qu'irréel de la mort/Mort, Holbein lui donne une puissance immense, pour la transmuer en même temps en fait mental ; jouant consciemment (comme quelques décennies plus tard Bruegel) avec les différentes notions de la mort/Mort, il lui donne l'existence insaisissable, l'identité fluctuante du double. Il trace des scènes où la figure de la Mort ne renvoie qu'à la mortalité générale des humains, d'autres où l'agissement violent des squelettes marque l'instant final d'une personne et encore d'autres où le squelette signifie un événement naturel (tel que le mauvais temps qui fait chavirer le bateau ; $\mathrm{n}^{\circ} 30$; fig. $6 \mathrm{e}$ ). Une gravure, ajoutée dans l'édition augmentée que Bruegel connaissait probablement, identifie la figure de la Mort avec la guerre ( $\mathrm{n}^{\circ} 40$; fig. $6 \mathrm{~g}$ ) : tandis qu'un guerrier combat au premier plan avec un squelette, un autre squelette mène, en arrière-plan, l'armée sur le champ de bataille qui est déjà recouvert de morts et de mourants ${ }^{88}$. Ainsi, la Mort est devenue une figure polémique ${ }^{89}$, le représentant d'une propagande contre la guerre telle qu'elle était déjà inaugurée dans quelques gravures d'Urs Graf ${ }^{90}$ avant qu'elle ne trouve l'expression la plus forte chez Bruegel.

86. M. Foucault, op. cit., p. 20.

87. K. HOFFMANN, op. cit., p. 264 ss.

88. F. BÄCHTIGER, op. cit., p. 142 ss.

89. Voir F. Petersmann, Kirchen- und Sozialkritik in den Bildern des Todes von Hans Holbein d. J., Bielefeld, 1983 ; K. HoffMANN, op. cit., montre que déjà l'Alphabet de la Mort, conçu d'abord comme tract, se faisait comprendre comme satire morale au sens de la Nef des fous (p. 269).

90. F. BäCHTIGER, op. cit., p. 136 ss ; St. KOZÁKy, op. cit., ill. V/1 ; Himmel, Hölle, Fegefeuer, op. cit., p. 258 ss (ill.). 


\section{KIENING}

Qui volunt diuites fieri incidunt in laqueum diaboli, $\&$ defideria multa, $\&$ nociua, quas mergunt homines in interitum.

I I D TIMO. VI

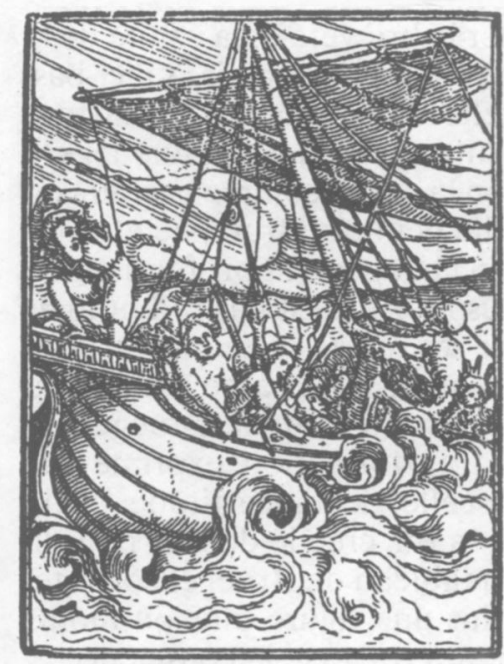

Pour acquerir des biens mondains Vous entrez en tentation, Qui uous met es perilz foubdains, Et wous maine a perdition.

$F$ iï

FIG. 6 suite. - Holbein/Lützelburger, Images de la Mort, gravures sur bois; Les simulachres \& historiees faces de la mort, Lyon : Trechsel, 1538 (d'après le fac-similé, New York, 1971).

e, ci-dessus à gauche. La tempête (p. 45 ; $\mathrm{n}^{\circ} 30$ ).

f, ci-dessus à droite. Le blason de la Mort (p. $\left.56 ; \mathrm{n}^{\circ} 41\right)$.

$\mathrm{g}$, ci-contre. La Mort et la guerre (ajouté en $n^{\circ} 40$ dès l'édition de 1545).
RENCONTRES DES VIVANTS ET DES MORTS

\section{Memorare nouiffima, \& in zeternum non pece. cabis.}

E C C L B. VII

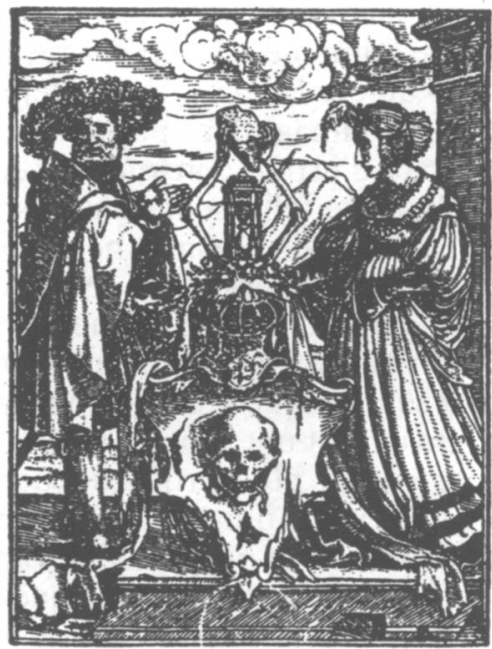

Si tu ueulx uiure fans peché Voy cefte imaige a tous propos, Et point ne feras empefché, Quand tu t'en iras a repos.

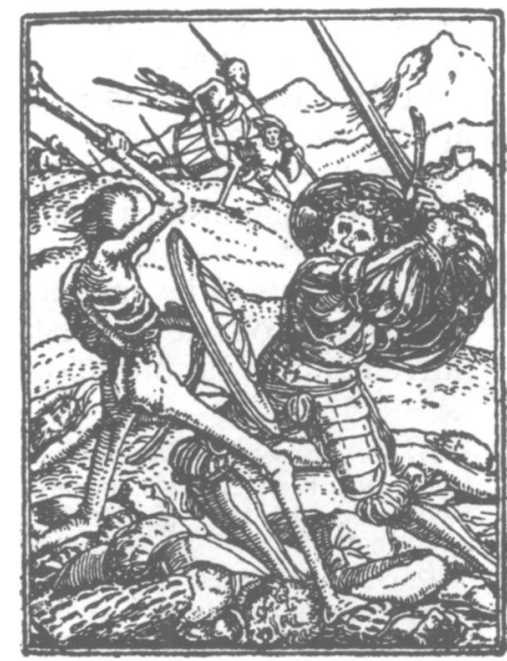




\section{IMAGES DE LA MORT, DESTRUCTION DES IMAGES AU MOYEN AGE}

En parallèle avec l'augmentation de la vitalité des morts/Morts, un accroissement de la résistance des vivants est mis en scène. Contre la force anarchique et archaïque attribuée aux «autres », ils commencent à développer une volonté de vivre qui, mise en évidence par la terreur que les hommes expriment chez Holbein, ne s'accorde plus avec la conception d'une bonne mort chrétienne. La danse macabre, inventée pour articuler la brièveté et la vanité de la vie humaine, se transforme ainsi paradoxalement en aide à vivre dans l'ici-bas. D'ailleurs, dans le même temps des formes nouvelles se développaient, surtout dans l'imaginaire populaire, qui rend à la rencontre avec la Mort une légère trace d'espoir : on joue aux échecs avec la Mort, on la prend comme parrain, on l'intègre tellement dans la sphère humaine qu'elle peut être trompée et vaincue $^{91}$. Ces fantasmes ne sont que les fleurons d'un changement profond qui se manifeste aussi là où le pouvoir de la Mort et des morts n'est pas réduit mais augmenté. Même si chez Bruegel la lutte contre les morts reste sans espoir, l'évidence de la mort est devenue ambivalente - justement parce que le flou " sémantique » d'une conception de la mort est devenu évident. Bruegel, en inventant une «boîte noire » en tant que lieu de transformation, exprime une angoisse plus profonde que celle de mourir: celle de l'incertitude de la conscience qui, ne pouvant pas vraiment penser sa fin, crée le double pour la penser - et qui recule devant lui. La subtilité du tableau de Bruegel consiste, semble-t-il, dans le fait que le double n'y est plus une figure plus ou moins concrète, mais justement la déchirure, la brisure qui traverse la conscience.

Arrivé à ce point, on aperçoit mieux et les héritages et les innovations dans les conceptions de Holbein et Bruegel en rapport avec l'immense multiplication des cadavres, des squelettes et des Morts personnifiés aux $15^{\mathrm{e}}$ et $16^{\mathrm{e}}$ siècles. Les textes et les images, bien qu'ils expriment le problème de la mortalité humaine et particulièrement celui de la mors improvisa, bien qu'ils fussent toujours de nouveau utilisés pour les intentions du clergé, ne se laissent pas réduire à leur fonction de rappe ${ }^{92}$. Dans l'égalité qui est affichée par les danses macabres, par exemple, se révèle aussi une critique sociale qui peut être tournée contre les auteurs cléricaux et les ordres mineurs euxmêmes, se dévoile une joie sadique qui peut saper l'intention chrétienne et qui peut, par des curieux fantasmes de survie en forme de mort-vivant, transmettre des impulsions non voulues pour redéfinir la corporalité et l'existence terrestre.

Les textes et les images dits «macabres » tournent plus ou moins explicitement autour de la question qui ne pouvait pas non plus être résolue définitivement par la sotériologie chrétienne : comment vivre face à la mortalité,

91. O. Schwebel, Der Tod in deutscher Sage und Dichtung, Berlin, 1876; I. Müller et L. RÖHRICH, «Deutscher Sagenkatalog X : Der Tod und die Toten », Deutsches Jahrbuch für Volkskunde, 13, 1967, pp. 346-397 ; J. MARKALE, Contes de la mort des pays de France, Etrépilly, 1986.

92. Sur les images de la Mort dans le contexte du contemptus mundi, voir C. KIENiNG, "Contemptus mundi in Vers und Bild am Ende des Mittelalters ", Zeitschrift für deutsches Altertum und deutsche Literatur, 123, 1994, pp. 409-457, 482. 
à la dégradation du corps et à l'anéantissement de la mémoire, face aux tensions entre un passé surpuissant, incarné par les morts, et un avenir incertain. Les réponses données avec une subtilité croissante ne représentent, pour la plupart, ni des réactions directes à quelques événements historiques (catastrophes épidémiques), ni de simples jeux futiles. Ils témoignent plutôt des angoisses et des préoccupations, dont ils font l'expérience dans le même temps. Les miroitements observés au-dessus ne se limitent pas aux rapports internes des images. La gravure finale de Holbein aussi bien que le type du «double portrait macabre ${ }^{93}$ indiquent une relation intime entre le spectateur et son «image », une méditation personnelle à travers la figure qui représente le "propre » et l'« autre » de l'existence humaine ${ }^{94}$. Les images (plus subtiles que les textes) projettent la possibilité d'une «contre-force » qui remet l'homme et la vie en question, mais qui se focalise aussi, justement à partir du point limite de la non-existence, sur le sens et la valeur de la vie.

Le thème de la mort et du double s'y joint au thème de l'individualité et demande l'intégration dans une perspective historique plus nuancée et plus large ${ }^{95}$. C'est non seulement, d'après les analyses de Philippe Ariès ${ }^{96}$, la «mort de soi », que l'homme occidental aurait découvert dans un long processus dès le $12^{\mathrm{e}}$ siècle, mais c'est aussi la «mort du Soi » (et avec celle-ci le Soi lui-même) qui attirait dès lors l'attention. L'attitude face à la mort/Mort s'associe ainsi à un discours de la personne aussi bien qu'à un discours de l'altérité qui, tous les deux, sont en train de redéfinir l'auto-perception de l'homme occidental à la fin du Moyen Age.

Les expérimentations avec les possibilités iconographiques de la figure squelettique n'y forment que l'aspect d'un univers complexe, mais aussi (au

93. Voir E. BuCHNER, Das deutsche Bildnis der Spätgotik und der frühen Dürerzeit, Berlin, 1957 ; J. WIRTH, op. cit., p. 38 ss.

94. Le meilleur exemple pour le rôle constitutif du spectateur est le fameux tableau de Holbein de 1533 : Les ambassadeurs, destiné probablement au château de Jean de Dindeville (l'un des deux ambassadeurs) à Polisy (aujourd'hui : Londres, National Gallery). Holbein y a placé entre les deux ambassadeurs une tête décharnée en forme d'anamorphose qui n'est apercevable sans distorsion que de l'angle droit du tableau, d'où, en revanche, le reste du tableau n'est plus visible. Ainsi, la vie et la mort, le savoir et son anéantissement se trouvent sur des plans perspectifs différents et ne peuvent être aperçus que successivement. Une interprétation convaincante du tableau est donnée par K. HoffmanN, "Hans Holbein d. J. : Die "Gesandten" ", Festschrift für Georg Scheja zum 70. Geburtstag, A. LeUTERITz et al., éds, Sigmaringen, 1975, pp. 133-150, qui se refère aux œuvres de Georges de Selve (l'autre « ambassadeur ») ; voir aussi St. Greenblatt, Renaissance Self-Fashioning: From More to Shakespeare, Chicago, 1980, pp. 17-26.

95. Voir K. HoffMANN, « Angst und Gewalt als Voraussetzungen des nachantiken Todesbildes ", Angst und Gewalt. Ihre Präsenz und Bewältigung in den Religionen, H. vON STIETENCRON éd., Düsseldorf, 1979, pp. 101-110, part. 109: «Die Entstehung des neueren Todesbildes fällt in den Zeitraum, der nach der historischen Verhaltensforschung gekennzeichnet ist durch den zunehmenden gesellschaftlichen Zwang zu individuellem Selbstzwang. Auf der einen Seite steht die Herausbildung von "Staat" als kollektiver Monopolisierung von Gewalt, auf der anderen Seite die Individualisierung, auch von Todesangst »; en outre A. HAHN, « Tod und Individualität. Eine Übersicht über neuere französische Literatur », Kölner Zeitschrift für Soziologie, 31, 1979, pp. 746-765.

96. P. ARIÈs, Essais sur l'histoire de la mort en Occident du Moyen Age à nos jours, Paris, 1975, pp. 37-50. 


\section{IMAGES DE LA MORT, DESTRUCTION DES IMAGES AU MOYEN AGE}

moins en surface) uniforme. Car, comme une métaphore perd son acuité, les figurations de la Mort - dont d'ailleurs un nombre considérable s'insère parfaitement dans la pensée chrétienne de la mort comme intermédiaire, associé au péché originel - peuvent se changer en formes vides. Dès la deuxième moitié du $15^{\mathrm{e}}$ siècle, la Mort, libérée des contextes bien déterminés, apparaît partout : dans des gravures qui stigmatisent la prostitution ${ }^{97}$ aussi bien que dans celles qui illustrent le combat pour un pantalon ${ }^{98}$. La figure squelettique est devenue un signe aussi familier que flou pour la vanité, un signe qui, réduit jusqu'au pur emblème du crâne, apaise la conscience plus qu'il ne la trouble. Mais elle garde un potentiel bouleversant qui peut toujours de nouveau s'épanouir et mettre en jeu les ambivalences psychiques face à la mort que Vauzelles, dans la préface des Simulachres, a saisies, quand il promettait « que lon y peut prendre vne delectable tristesse, $\&$ vne triste delectation, comme en chose tristement ioyeuse » (Aiiiv).

Christian KIENING Université de Munich

97. Vienne, Albertina ; W. Mezger, Narrenidee, op. cit., p. 439 ss et ill. 237.

98. Munich, Staatl. Graphische Sammlung, Inv. Nr. 174027 (Die Graphiksammlung des Humanisten Hartmann Schedel [catalogue : B. HeRNAD], Munich, 1990, p. 284 ss et ill. 30). 